Journal of Management

Vol. 42 No. 5, July 2016 1195-1229

DOI: $10.1177 / 0149206316632058$

(C) The Author(s) 2016

Reprints and permissions:

sagepub.com/journalsPermissions.nav

\title{
A Review of Self-Determination Theory's Basic Psychological Needs at Work
}

\author{
Anja Van den Broeck \\ KU Leuven \\ North-West University \\ D. Lance Ferris \\ The Pennsylvania State University \\ Chu-Hsiang Chang \\ Michigan State University \\ Christopher C. Rosen \\ University of Arkansas
}

Self-determination theory (SDT) conceptualizes basic psychological needs for autonomy, competence, and relatedness as innate and essential for ongoing psychological growth, internalization, and well-being. We broadly review the literature on basic psychological need satisfaction at work with three more specific aims: to test SDT's requirement that each basic psychological need should uniquely predict psychological growth, internalization, and well-being; to test whether use of an overall need satisfaction measure is appropriate; and to test whether the scale used to assess basic psychological needs influenced our results. To this end, we conducted a meta-analytic review of 99 studies with 119 distinct samples examining the antecedents and consequences of basic need satisfaction. We conclude with recommendations for addressing issues arising from our review and also identify points for future research, including the study of need frustration and culture, integrating the basic needs with other motivation theories, and a caution regarding the measures and methods used.

Keywords: needs; motivation; meta-analysis; review; self-determination theory

\footnotetext{
Acknowledgments: We would like to dedicate this manuscript to Willy Lens. We thank Daniel Beal and two anonymous reviewers for their helpful comments and advice in the review process. A portion of the work on this manuscript was completed while the fourth author was a visiting faculty member at Texas Christian University.

Supplemental material for this article is available at http://jom.sagepub.com/supplemental

Corresponding author: Anja Van den Broeck, Faculty of Economy and Business, KU Leuven, Warmoesberg 26, 1000 Brussels, Belgium.
}

E-mail: anja.vandenbroeck@kuleuven.be 
Motivation, or the "energetic forces that initiate work-related behavior and determine its form, direction, intensity and duration" (Pinder, 2008: 11), is a critical issue for organizations and employees. It has been linked to increased employee productivity and organizational revenue, as well as employees' well-being and thriving (Steers, Mowday, \& Shapiro, 2004). Given its important role, a good deal of research has focused on the type and extent of motivation employees experience (e.g., Diefendorff \& Chandler, 2011; Latham \& Pinder, 2005). Within this research area, a prominent focus has been on how the satisfaction of needs, or "some type of internal tension or arousal" (Kanfer, 1990: 81), enhances employee motivation. For example, needs have figured in historical frameworks from Maslow's (1943) need hierarchy to McClelland's (1965) work on needs for achievement, affiliation, and power. More recently, researchers have proposed alternate needs, such as the need for status (Hogan, 1998) and the need for relatedness (Baumeister \& Leary, 1995; Deci \& Ryan, 2000).

Yet few need frameworks have spurred as much research on needs as self-determination theory (SDT; Deci \& Ryan, 2000). SDT argues that humans are optimally motivated and experience well-being when they have three basic psychological needs satisfied: the need for autonomy, the need for competence, and the need for relatedness (Deci \& Ryan). Basic psychological needs have been the focus of research in numerous domains, such as education (Vansteenkiste, Lens, \& Deci, 2006), health care (Ng et al., 2012), and sports and exercise (Edmunds, Ntoumanis, \& Duda, 2006). Within the domain of organizational research, basic psychological needs have been used across a variety of topics, including leadership (Lian, Ferris, \& Brown, 2012), organizational politics (Rosen, Ferris, Brown, Chen, \& Yan, 2014), employee well-being (Deci, Ryan, Gagné, Leone, Usunov, \& Kornazheva, 2001), person-environment fit (Greguras \& Diefendorff, 2009), job design (Van den Broeck, Vansteenkiste, De Witte, \& Lens, 2008), and proactive personality (Greguras \& Diefendorff, 2010), among others.

The increasing importance and popularity of basic psychological needs in the organizational domain, combined with the lack of any existing reviews on the topic, highlights the need for a conceptual and empirical review of the management research on this topic. ${ }^{1}$ To this end, our paper provides a meta-analytic overview of organizational research on basic psychological needs, demonstrating the breadth of constructs (i.e., antecedents and consequences) that basic psychological needs have been found to relate to. We also had three more specific aims with our review: to test SDT's requirement that each basic psychological need should uniquely predict psychological growth, internalization, and well-being; to test whether use of an overall need satisfaction measure is appropriate; and to test whether the scale used to assess basic psychological needs (i.e., the measure developed by Deci et al., 2001, or by Van den Broeck, Vansteenkiste, De Witte, Soenens, \& Lens, 2010) influenced our results.

In accomplishing these aims, our review provides both contributions and challenges to the SDT literature. In particular, our results indicate research on basic psychological needs is both vibrant and prolific, with need satisfaction relating to a wide variety of antecedents and outcomes. Moreover, we find general support for SDT's requirement that each need should independently predict indicators of psychological growth, internalization, and well-being. However, our findings also illustrate that contrary to SDT, the variance basic psychological needs account for in some of these outcomes is statistically significant but practically insignificant; our review also surfaces issues with the literature's most commonly used scale. 
In what follows, we first discuss SDT and its focus on psychological growth, internalization, and well-being and how SDT characterizes basic psychological needs as innate factors that are necessary for such outcomes to occur. We next discuss how this characterizationunique to SDT - helps differentiate SDT from other need theories. We then provide a metaanalytic review of the literature and summarize how our results provide both support for and challenges to SDT's view of basic psychological needs. Finally, we provide recommendations and new research directions to help address gaps and problems in the literature.

\section{SDT and Basic Psychological Needs}

SDT starts from the premise that the natural inclination and progression of humans is towards psychological growth, internalization, and well-being and that humans act on-and are acted upon by - the environment in ways that differentially facilitate or hinder the realization of this natural progression (Deci \& Ryan, 2000). Given these natural inclinations towards psychological growth, internalization, and well-being, these three outcomes are frequently the criterion variables of interest in SDT research, with each operationalized in different ways. Psychological growth is typically manifested by intrinsic motivation, or the curious and exploratory engagement in activities that individuals find inherently interesting and enjoyable and that are done even in the absence of external reinforcement (Deci \& Ryan, $1980,2000)$. The use of the "psychological growth" term when referring to intrinsic motivation stems from the belief in SDT that intrinsically motivated individuals are "involved in an ongoing, cyclical process of seeking out (or creating) optimally challenging situations and then attempting to conquer those challenges"-or put differently, intrinsic motivation leads to the psychological growth of the individual (Deci \& Ryan, 1980: 42).

Psychological internalization represents the natural inclination for individuals to transform external reasons for engaging in a behavior into forms of motivation that are more fully internalized and integrated within the self (Deci \& Ryan, 1985). More specifically, SDT recognizes that extrinsic motivation - or engaging in a behavior for reasons other than the behavior being inherently interesting and enjoyable — can be operationalized in terms of three types of extrinsic motivation: (a) external motivation, in which behavior is engaged in because individuals feel forced to do so because others provide external punishment/rewards for engaging (or not engaging) in the behavior; (b) introjected motivation, in which behavior is engaged in because individuals would feel pride, shame, or guilt if they engaged (or did not engage) in the behavior; and (c) identified motivation, in which behavior is more selfendorsed and viewed as important and/or in line with one's closely held values. ${ }^{2}$ External and introjected motivation are both controlled, as they pertain to external or internal pressure, whereas identified and intrinsic motivation imply the endorsement of the reasons behind one's behavior and are therefore autonomous. The main difference between identified and intrinsic motivation is that with identified motivation, the behavior engaged in is not considered enjoyable in and of itself: For example, if professors dislike teaching but nevertheless put effort into crafting their courses because being an educator is a key part of their self-view, they possess identified motivation; professors possess intrinsic motivation if they find crafting courses to be enjoyable in and of itself. Notably, employees can possess multiple motivation forms for engaging in a given behavior, as, for example, professors may put effort in their teaching both because they enjoy it (i.e., intrinsic motivation) and because they see it as 
needed for tenure (i.e., external motivation; see also Moran, Diefendorff, Kim, \& Liu, 2012; Van den Broeck, Lens, De Witte, \& Van Coillie, 2013).

Finally, psychological well-being is typically operationalized in SDT research using measures drawn from hedonic and eudaemonic well-being perspectives (Ryan \& Deci, 2001). This includes measures such as experienced positive and negative affect, life satisfaction, mental and physical health, and vitality.

\section{Basic Psychological Needs and Psychological Growth, Internalization, and Well-Being}

While SDT argues that all individuals possess this natural inclination towards psychological growth, internalization, and well-being, it also acknowledges that the inclination is not always expressed or achieved: Individuals may behave passively, and they may engage in counterproductive behaviors that ultimately thwart growth, internalization, or well-being (Ryan \& Deci, 2000; Vansteenkiste \& Ryan, 2013). Whether individuals realize their natural tendencies depends on whether individuals experience what SDT considers to be the fundamental nutriments required to achieve these tendencies. In particular, just as plants need water, sunshine, and minerals to thrive, SDT argues that the satisfaction of three basic psychological needs for autonomy, competence, and relatedness are essential for individuals to achieve psychological growth, internalization, and well-being (Deci \& Ryan, 2000). Specifically, having one's needs satisfied leads to more autonomous forms of motivation (i.e., identified and intrinsic motivation) and improved mental health and well-being. In this sense, basic psychological needs are arguably the most important constructs within SDT (Ryan \& Deci).

SDT defines the need for autonomy as individuals' need to act with a sense of ownership of their behavior and feel psychologically free (Deci \& Ryan, 2000). The need for autonomy draws from the notion of locus of causality, or being the origin of one's actions rather than being pushed and pulled around by external forces (deCharms, 1968). The need for autonomy was the focus of early SDT research, as it proved to be essential in explaining the negative impact of extrinsic incentives on the emergence and sustainability of intrinsic motivation (Deci, Ryan, \& Koestner, 1999). Of the three basic psychological needs, the need for autonomy remains among the most controversial, although this is primarily due to misunderstanding over the nature of the need (Deci \& Ryan). In particular, the need for autonomy does not imply a need to act independently from the desires of others; rather, it implies the need to act with a sense of choice and volition, even if doing so means complying with the wishes of others. For example, a manager may ask an employee to complete a particular task during a lunch break; if the employee volitionally agrees to do so, the need for autonomy is satisfied. On the other hand, if the employee would rather go out for lunch and feels forced to keep working, autonomy will be thwarted (Trougakos, Hideg, Cheng, \& Beal, 2013).

Compared with autonomy, the other two basic psychological needs are less controversial or misunderstood. Following White (1959), SDT defines the need for competence as the need to feel a sense of mastery over the environment and to develop new skills. The need for competence originally became a focus of SDT research as researchers sought to explain how verbal praise could still enhance intrinsic motivation, despite its extrinsic nature (Deci et al., 1999); now, SDT views the need for competence as inherent to our natural tendency to 
explore and manipulate the environment, as well as in the search for optimal challenges. The need for competence also figures in other theories, such as social cognitive theory, where self-efficacy is considered the primary motivational principle (Bandura, 1977).

The final and most recent addition to the basic psychological needs category is the need for relatedness. The need for relatedness represents the need to feel connected to at least some others, that is, to love and care for others and to be loved and cared for by others (see also Baumeister \& Leary, 1995). This need is satisfied when people see themselves as a member of a group, experience a sense of communion, and develop close relations. The inclusion of relatedness as a basic psychological need was grounded in its evolutionary benefits in terms of survival and reproduction. The need for relatedness is sometimes characterized as being less immediately essential for some outcomes than the needs for autonomy or competence. For example, a child may intrinsically enjoy playing with toys alone, meaning the activity itself does not satisfy the need for relatedness. Nevertheless, SDT argues such intrinsic motivation could not emerge in the absence of secure relational attachments (e.g., to parents; Deci \& Ryan, 2000).

\section{Differences Conceptualizing Needs in SDT and Other Theories}

As noted previously, the concept of needs and need satisfaction is not new within the motivation literature, with researchers postulating various need candidates over the decades. At the same time, SDT characterizes basic psychological needs in two ways that render it unique in comparison to other need theories: needs are viewed as innate, and needs must promote psychological growth, internalization, and well-being (Deci \& Ryan, 2000).

First, within SDT, needs are conceptualized as innate fundamental propensities all individuals possess (Ryan \& Deci, 2000), much like physiological needs, such as hunger and thirst (Hull, 1943). In this sense, SDT differs from other need theories, such as McClelland's (1965) acquired needs theory, which argues the needs for achievement, power, and affiliation are acquired via socialization and learning throughout the life span (see also Murray, 1938). As a result, from McClelland's perspective, individuals should differ in which needs are present or which dominate, while in SDT, each need is thought to be present in everyone, and none of the needs are thought to be relatively more important than the others. SDT thus regards each of the three needs as essential, with thwarting of any one need causing disruptions to psychological growth, internalization, and well-being. This contrasts with other need theories that argue for a hierarchy of needs - the most famous being that of Maslow (1943), who argued that needs higher in his hierarchy become more activated when needs at the bottom of the hierarchy are satisfied. As a result of viewing basic psychological needs as being innate, SDT research tends to focus on need satisfaction rather than need strength. That is, some need theories focus on how strongly an individual has, for example, a need for power or affiliation (McClelland; Murray). While SDT does not rule out that individuals may differ in the strength of the desire for the needs, even those who express a weak desire for a given need are nevertheless argued to benefit from the satisfaction of that need (Deci \& Ryan, 2000). As a result, SDT research has generally not examined moderators (such as indices of need strength) of the effects of need satisfaction.

Second, SDT is perhaps unique among need theories in that it provides objective criteria for why some constructs but not others should be considered "basic psychological needs." 
Within SDT, basic psychological needs are those critical conditions that enable the expression of our natural inclinations towards psychological growth, internalization, and well-being (Deci \& Ryan, 2000: 229). Basic psychological needs in SDT are thus primarily determined via inductive processes: Constructs are classified as needs when enough evidence exists to suggest satisfaction of the need contributes to psychological growth, internalization, and well-being over and above other established needs.

In defining needs in this fashion, SDT differentiates "needs" from what might be referred to as "desires" (Deci \& Ryan, 2000). People may desire power, money, status, fame, or to be beautiful, but they do not "need" it in an SDT sense; for example, not everybody expresses a need for power, and its presence or absence may not contribute to intrinsic motivation, or individuals' ability to internalize external motivation within their sense of self, or well-being (Deci \& Ryan; Greguras \& Diefendorff, 2009). Indeed, research suggests that people typically do not experience well-being when they have a strong need for concepts such as power or wealth (Sheldon \& Kasser, 1998). Likewise, although exercising may lead to well-being (and for some it may even be an activity that people are intrinsically motivated to do), it is unlikely to foster psychological internalization.

By requiring that different "needs" should predict psychological growth, internalization, and well-being over and above the effects of other basic needs, a high standard is set for any potential new need to be added to the theory. In particular, any potential need candidate must consistently continue to predict psychological growth, internalization, and well-being across multiple samples, even once the effects of autonomy, competence, and relatedness are accounted for. Interestingly, despite the general prominence of need theories in the management literature, systematic comparisons of alternate need candidates (e.g., for status or power) against the effects of basic psychological needs has generally not been conducted in organizational research (but see Sheldon, Elliot, Kim, \& Kasser, 2001, for a nonorganizational example).

\section{Measuring Basic Psychological Needs at Work}

To date, several measures have been developed to operationalize basic need satisfaction at work, with published work typically using the Basic Need Satisfaction at Work scale. This 21-item questionnaire builds on early research on basic psychological needs (e.g., Ilardi, Leone, Kasser, \& Ryan, 1993) and was further developed for management research (Baard, Deci, \& Ryan, 2004; Deci et al., 2001). Although widely used, this questionnaire has been criticized for a number of reasons. First, the scale was not stringently validated, and subsequent research has reported problems with the reliability of and high intercorrelations among the subscales (e.g., Gagné, 2003; Greguras \& Diefendorff, 2009). Second, questions have been raised regarding the content validity of the scale (Van den Broeck et al., 2010), as some items assess antecedents of need satisfaction (e.g., job autonomy: "I feel like I can make a lot of inputs to deciding how my job gets done"; or positive feedback: "People at work tell me I am good at what I do"), while other items assess the consequences of basic need satisfaction (e.g., intrinsic motivation: "I enjoy the challenge my work provides").

More recently, the Work-Related Basic Need Satisfaction measure has been developed (Van den Broeck et al., 2010). Following traditional validation processes (e.g., Hinkin, 1998), the reliability of the basic needs subscales and a tripartite factor structure has been 
established. To avoid content validity issues, Van den Broeck et al. designed the scale such that it directly assesses satisfaction of the needs for autonomy (e.g., "The tasks I have to do at work are in line with what I really want to do"), competence (e.g., "I feel competent at my job"), and relatedness (e.g., "At work, I feel part of a group") rather than their antecedents or consequences.

\section{Meta-Analysis of the Basic Need Satisfaction Literature}

As noted at the outset of our paper, research linking basic psychological need satisfaction in the workplace to various organizational concepts has increased, particularly over the last 15 years. Thus, one purpose of our review is to provide a basic overview of the various workplace antecedents and consequences that have been linked to workplace need satisfaction. In doing so, our review provides researchers a summary of where SDT research has been, as well as benchmarks for correlational effect sizes for future research. Beyond providing this overview, our review had three more specific aims.

The first aim was to test SDT's primary criteria for calling autonomy, competence, and relatedness "basic psychological needs," that is, that each of the three needs for autonomy, competence, and relatedness should demonstrate incremental predictive validity in the prediction of measures of psychological growth, internalization, and well-being, even when controlling for the effects of the other two needs. Although this represents the primary criteria for being a "basic psychological need," tests of this requirement have largely been overlooked in organizational contexts. Following SDT, we operationalized psychological growth with measures of intrinsic motivation; psychological internalization with measures of external motivation, introjected motivation, and identified motivation (as well as measures of amotivation, or a lack of either extrinsic or intrinsic motivation); and psychological wellbeing using a number of common measures of well-being, including positive affect, negative affect, and life satisfaction, as well as other measures tapping into work-related well-being and physical and psychological strain.

The second aim was to test whether averaging the needs into an overall need satisfaction measure is appropriate. Though it may seem self-evident that, for example, the need for autonomy is not the same as the need for relatedness, the three needs are often averaged into a single score to assess overall need satisfaction (e.g., Deci et al., 2001; Lian et al., 2012). This has been justified by arguments that the needs load on a single factor (Van den Broeck et al., 2008), are highly correlated (Gagné, 2003), or share nomological networks (Rosen et al., 2014). However, if - as SDT argues - each need represents an independent construct, averaging the needs to create an index of overall need satisfaction is inappropriate because it treats each need as interchangeable and assumes low satisfaction of autonomy can be rectified by high satisfaction of competence, which SDT specifically argues is not the case.

To evaluate the appropriateness of averaging the three needs into an overall need satisfaction measure, we used three criteria. The first criterion was the correlations among the three needs; in general, correlations exceeding .70 indicate that two constructs may represent the same construct and be interchangeable, as this is the minimum correlation required to establish split-half reliability of measures assessing one construct (Nunnally, 1967). The second criterion was the results of relative weight (RW) analyses we conducted to examine the incremental predictive validity of each need when predicting outcomes in the SDT 
literature - specifically, measures of motivation and well-being (as noted above), but also including job attitudes and job behaviors. Demonstrating incremental predictive validity in these analyses suggests the measures are not redundant or interchangeable with each other (given that redundant measures would not incrementally predict beyond each other) and so should be considered separately. The third criterion was to examine whether the nomological networks of the antecedents of need satisfaction were similar; if the confidence intervals for the correlation of each need with a given antecedent do not overlap, this would suggest that the relations are different and the needs should not be combined together in an overall score. To test this, we examined the relation of the needs with various antecedents (e.g., individual differences, job resources, job stressors).

Finally, the third aim was to test whether the effects of need satisfaction varied across the measure used. As noted previously, prior work has suggested potential problems with the dominant measure used to assess need satisfaction at work (i.e., the Deci et al., 2001, scale), including poor reliability and high intercorrelations among the needs. Thus, where possible, we examined whether our findings (including the relations of the needs with antecedents, consequences, and each other) differed depending on whether the Deci et al. scale or the more newly developed scale (i.e., the Van den Broeck et al., 2010, scale) was used.

\section{Literature Search}

To search for studies, between approximately August 2014 and January 2015, we conducted independent searches of four databases: PsycINFO, Web of Science, ProQuest for interdisciplinary dissertations and theses, and Google Scholar. We used keywords associated with SDT needs, including "need satisfaction," "basic needs," and "needs + self determin*," which were paired with "employ*" or "work*" for the database search. We placed no date, geographical, cultural, language, or population restrictions on the search. Using the same databases, we then searched all articles citing well-established papers (Baard et al., 2004; Deci et al., 2001) and scale validation studies (Van den Broeck et al., 2010). We also posted calls for unpublished papers on the listservers of the Organizational Behavior and Human Resources Divisions of the Academy of Management, as well as the SDT Web site listserver. Finally, we contacted active SDT researchers for unpublished studies we may have missed. In total, we identified 99 relevant papers with 119 separate samples that could be included in the meta-analysis. References for the data used in the meta-analysis can be found in the online supplemental material.

\section{Inclusion Criteria and Coding}

Empirical studies were selected for inclusion in the meta-analysis if they fit three criteria. First, studies had to examine adult participants in an organizational setting. Second, only empirical studies that investigated relationships between at least one need and at least one antecedent or outcome measure were included. Third, only empirical studies that allowed us to gather correlations for each need separately were included. When a paper was missing this information, we contacted the paper's corresponding authors to request the information.

Once papers were selected for inclusion, the first, second, and fourth authors split up and entered the data for each study into an overall spreadsheet; the third author reviewed the data. 
Any discrepancies were resolved through discussion among the authors. Although all study data were initially entered into the spreadsheet, we excluded from analysis any correlation between needs and a particular variable that did not occur in at least three samples. Finally, we coded the studies for the needs measure used and publication status.

Correlation coefficients were collected as effect sizes. When a study reported correlations between a need satisfaction measure and multiple measures of the same antecedent or outcome construct (e.g., emotional exhaustion, depersonalization, and reduced personal accomplishment for burnout), the effect sizes were averaged together and reported for the overall construct (in this example, burnout). In the online supplemental materials, estimates for specific breakdowns of constructs are provided (e.g., the estimates for the relation of each need to emotional exhaustion, depersonalization, and reduced personal accomplishment). This approach is commonly adopted to avoid inflation of the sample size (Cheung \& Chan, 2004). However, because this procedure does not take into consideration the level of dependence across effect sizes from the same sample, it likely underestimates the heterogeneity among these effect sizes. As such, the adjusted-weighted procedure (Cheung \& Chan) was used to calculate the adjusted sample size whenever multiple effect sizes from the same sample were averaged in order to account for the relatedness among these effect sizes. The adjusted sample size was then used as the sample weight for the sample-weighted average effect size.

\section{Procedure}

We conducted the meta-analysis following Arthur, Bennett, and Huffcutt's (2001) strategy, which is based upon the Hunter-Schmidt model. For each target relationship, we first calculated a sample-weighted mean correlation $(r)$. We computed the percentage of variance accounted for by sampling error (Hunter \& Schmidt, 2004) to indicate the sampling error associated with sample sizes. The chi-square test for the homogeneity was calculated (Rosenthal, 1991) to inform the estimation of standard error used to compute the $95 \%$ confidence interval around the sample-weighted mean correlation (Whitener, 1990). The confidence interval was used to determine whether the relationships between needs and antecedent or outcome measures were significantly different from 0 , such that a $95 \%$ confidence interval excluding 0 indicates that the correlation is significant. ${ }^{3}$

We then performed the statistical correction for attenuating artifacts (e.g., unreliability of measures from empirical studies; Hall \& Brannick, 2002) to derive the corrected estimate of correlation coefficient ( $\rho$; Hunter \& Schmidt, 2004). We then computed the variance and standard deviation of the corrected correlation following the random-effects meta-analysis strategy outlined by Hunter and Schmidt. The $Q$ statistic, which is based on a chi-square distribution, was calculated to determine whether differences among effect sizes between studies were significant (Sagie \& Koslowsky, 1993). Additional subgroup analyses were performed to examine the effects of a priori moderation effects associated with specific study characteristics (Cortina, 2003) with a significant $Q$ statistic. $Z$ tests that were based on the corrected correlation coefficient estimates and the pooled standard deviations of the mean of rho for each subgroup were conducted to compare the magnitude of relationships to test for moderating effects of study characteristics. Finally, to assess possible publication biases, we conducted subgroup comparisons between published versus unpublished studies: If an article was published or in press at the time that we conducted the meta-analysis, it was coded as 
Table 1

Meta-Analytic Relationships Between Individual Needs

\begin{tabular}{|c|c|c|c|c|c|c|c|c|c|c|}
\hline \multirow[b]{2}{*}{ Variables } & \multirow[b]{2}{*}{$k$} & \multirow[b]{2}{*}{$N$} & \multirow[b]{2}{*}{$r$} & \multirow[b]{2}{*}{$\rho$} & \multirow[b]{2}{*}{$S D \rho$} & \multirow[b]{2}{*}{$\% S E$} & \multicolumn{2}{|c|}{$95 \% \mathrm{CI}$} & \multirow[b]{2}{*}{$Q$} & \multirow[b]{2}{*}{ Tolerance } \\
\hline & & & & & & & Lower & Upper & & \\
\hline Autonomy - Competence & 105 & 45,824 & .44 & .57 & .21 & 5.19 & .41 & .47 & $902.16^{*}$ & 286,576 \\
\hline Competence - Relatedness & 104 & 45,698 & .35 & .45 & .21 & 5.73 & .32 & .39 & $1,201.60^{*}$ & 190,738 \\
\hline Autonomy - Relatedness & 104 & 45,702 & .47 & .61 & .14 & 9.15 & .45 & .50 & $476.36^{*}$ & 300,043 \\
\hline
\end{tabular}

Note: $k=$ number of effect sizes; $N=$ total subject number; $r=$ mean sample-weighted correlation; $\rho=$ estimate of corrected correlation; $S D \rho=$ corrected standard deviation of corrected correlation; $\% S E=$ percentage of observed variance accounted for by sampling error; $95 \% \mathrm{CI}=95 \%$ confidence interval around the mean sample-weighted correlation; $Q=$ chi-square test for the homogeneity of true correlations across studies; Tolerance $=$ number of studies showing null results necessary to eliminate the observed overall effect.

$* p<.05$.

published; others were coded as unpublished. We also estimated the tolerance value, or the number of studies showing null results that would be necessary to eliminate the observed overall effect (Rosenthal, 1979).

To test whether each of the three needs incrementally predicts outcomes, we conducted an RW analysis, which is a procedure that estimates the proportion of the total variance explained in an outcome variable $\left(R^{2}\right)$ that is attributable to each predictor (J. W. Johnson, 2000; LeBreton \& Tonidandel, 2008). Using corrected meta-analytic correlations and the corresponding total sample size $(N)$ from Tables 1, 5, and 6, we first calculated the harmonic mean of the sample size and then we ran the SPSS syntax developed by J. W. Johnson. In these multiple regression models, we reported the RW and the rescaled RW (i.e., RW divided by model $R^{2}$ ). Rescaled RWs represent the percentage of explained variance in an outcome variable that is attributable to each predictor. Results from examining the regression coefficients, RWs, and rescaled RWs indicate each need's relative importance in predicting the well-being and motivation outcomes.

\section{Meta-Analytic Results}

\section{Relations Among Needs}

Table 1 presents the corrected estimate of the population correlations ( $\rho$ ) among the basic psychological needs. Correlations among the needs are all positive and significant, with the correlations involving autonomy being the strongest.

\section{Relations Between Needs and Antecedent Variables}

The basic needs did not demonstrate a consistent pattern of relations with the demographic variables that were considered (see Table 2), as only 6 out of 15 of these relations were significant (all confidence intervals overlapped): Autonomy and competence demonstrated a positive relation with age and organizational tenure. Only relatedness was related to sex (women experienced more relatedness than men). The need for autonomy related positively to education. 
Table 2

Meta-Analytic Relationships Between Needs, Demographic Variables, and Individual Differences

\begin{tabular}{|c|c|c|c|c|c|c|c|c|c|c|}
\hline \multirow[b]{2}{*}{ Variables } & \multirow[b]{2}{*}{$k$} & \multirow[b]{2}{*}{$N$} & \multirow[b]{2}{*}{$r$} & \multirow[b]{2}{*}{$\rho$} & \multirow[b]{2}{*}{$S D \rho$} & \multirow[b]{2}{*}{$\% S E$} & \multicolumn{2}{|c|}{$95 \% \mathrm{CI}$} & \multirow[b]{2}{*}{$Q$} & \multirow[b]{2}{*}{ Tolerance } \\
\hline & & & & & & & Lower & Upper & & \\
\hline \multicolumn{11}{|l|}{ Autonomy } \\
\hline Age & 41 & 23,875 & .03 & .03 & .09 & 23.84 & .00 & .05 & $171.47 *$ & 1,822 \\
\hline $\begin{array}{l}\text { Tenure with } \\
\text { organization }\end{array}$ & 28 & 14,422 & .03 & .04 & .06 & 45.29 & .01 & .06 & $61.56^{*}$ & 482 \\
\hline $\begin{array}{c}\text { Tenure with } \\
\text { supervisor }\end{array}$ & 3 & 1,103 & .00 & .00 & .14 & 15.83 & -.14 & .15 & $18.95 *$ & 13 \\
\hline Sex & 42 & 25,272 & .01 & .01 & .04 & 56.39 & -.01 & .02 & $74.47^{*}$ & 687 \\
\hline Education & 22 & 7,541 & .09 & .10 & .11 & 24.63 & .04 & .13 & $86.84^{*}$ & 583 \\
\hline $\begin{array}{l}\text { Self-esteem and } \\
\text { efficacy }\end{array}$ & 11 & 3,209 & .35 & .47 & .15 & 15.00 & .27 & .42 & $40.35^{*}$ & 1,544 \\
\hline Optimism & 5 & 1,504 & .33 & .41 & .00 & 100.00 & .28 & .37 & 2.75 & 278 \\
\hline Mindfulness & 5 & 1,299 & .36 & .43 & .00 & 100.00 & .31 & .41 & 3.96 & 279 \\
\hline Agreeableness & 7 & 1,834 & .24 & .33 & .10 & 36.57 & .17 & .31 & $16.94 *$ & 253 \\
\hline Conscientiousness & 6 & 1,588 & .25 & .34 & .18 & 16.39 & .13 & .36 & $32.39 *$ & 196 \\
\hline Neuroticism & 3 & 879 & -.36 & -.47 & .18 & 12.30 & -.52 & -.19 & $21.91 *$ & 65 \\
\hline Extraversion & 3 & 879 & .20 & .27 & .05 & 71.90 & .14 & .26 & 4.05 & 27 \\
\hline Openness & 3 & 879 & .06 & .09 & .00 & 100.00 & -.00 & .13 & 0.54 & 1 \\
\hline Proactive personality & 4 & 660 & .26 & .32 & .00 & 100.00 & .19 & .33 & 1.77 & 62 \\
\hline Causality orientation & 5 & 1,132 & .20 & .32 & .11 & 40.53 & .11 & .29 & $10.03 *$ & 58 \\
\hline Extrinsic values & 7 & 3,564 & -.03 & -.04 & .04 & 68.20 & -.06 & .00 & 10.26 & 19 \\
\hline Intrinsic values & 9 & 4,333 & .18 & .23 & .00 & 100.00 & .15 & .21 & 6.58 & 373 \\
\hline \multicolumn{11}{|l|}{ Competence } \\
\hline Age & 41 & 23,875 & .05 & .06 & .12 & 13.95 & .02 & .08 & $291.46^{*}$ & 3,130 \\
\hline $\begin{array}{l}\text { Tenure with } \\
\text { organization }\end{array}$ & 28 & 14,419 & .08 & .09 & .06 & 38.88 & .05 & .11 & $70.38^{*}$ & 993 \\
\hline $\begin{array}{c}\text { Tenure with } \\
\text { supervisor }\end{array}$ & 3 & 1,103 & .02 & .03 & .05 & 53.97 & -.03 & .08 & 5.56 & 1 \\
\hline Sex & 42 & 25,269 & -.01 & -.02 & .07 & 27.91 & -.04 & .01 & $150.39 *$ & 1,617 \\
\hline Education & 22 & 7,541 & .02 & .02 & .12 & 20.84 & -.03 & .07 & $105.52 *$ & 447 \\
\hline $\begin{array}{l}\text { Self-esteem and } \\
\text { efficacy }\end{array}$ & 11 & 3,209 & .41 & .55 & .11 & 22.07 & .35 & .48 & $27.24 *$ & 2,071 \\
\hline Optimism & 5 & 1,504 & .35 & .43 & .00 & 100.00 & .31 & .40 & 1.34 & 336 \\
\hline Mindfulness & 5 & 1,299 & .39 & .47 & .05 & 56.27 & .34 & .43 & 7.50 & 324 \\
\hline Agreeableness & 7 & 1,834 & .25 & .35 & .08 & 47.49 & .19 & .32 & $12.64 *$ & 289 \\
\hline Conscientiousness & 6 & 1,588 & .38 & .51 & .08 & 37.13 & .31 & .45 & 10.84 & 463 \\
\hline Neuroticism & 3 & 879 & -.39 & -.47 & .10 & 26.16 & -.50 & -.28 & $9.50 *$ & 92 \\
\hline Extraversion & 3 & 879 & .33 & .42 & .06 & 48.61 & .25 & .41 & 5.41 & 64 \\
\hline Openness & 3 & 879 & .10 & .14 & .09 & 40.35 & .00 & .21 & $7.35^{*}$ & 15 \\
\hline Proactive personality & 4 & 660 & .34 & .42 & .00 & 100.00 & .27 & .41 & 1.01 & 107 \\
\hline Causality orientation & 5 & 1,132 & .18 & .25 & .00 & 100.00 & .12 & .24 & 1.03 & 54 \\
\hline Extrinsic values & 8 & 3,946 & .05 & .06 & .13 & 14.32 & -.03 & .13 & $55.66^{*}$ & 114 \\
\hline Intrinsic values & 9 & 4,329 & .15 & .19 & .11 & 19.78 & .09 & .22 & $43.72 *$ & 317 \\
\hline \multicolumn{11}{|l|}{ Relatedness } \\
\hline Age & 41 & 23,875 & -.02 & -.03 & .08 & 23.88 & -.05 & .00 & $171.60 *$ & 1,538 \\
\hline
\end{tabular}


Table 2 (continued)

\begin{tabular}{|c|c|c|c|c|c|c|c|c|c|c|}
\hline \multirow[b]{2}{*}{ Variables } & \multirow[b]{2}{*}{$k$} & \multirow[b]{2}{*}{$N$} & \multirow[b]{2}{*}{$r$} & \multirow[b]{2}{*}{$\rho$} & \multirow[b]{2}{*}{$S D \rho$} & \multirow[b]{2}{*}{$\% S E$} & \multicolumn{2}{|c|}{$95 \% \mathrm{CI}$} & \multirow[b]{2}{*}{$Q$} & \multirow[b]{2}{*}{ Tolerance } \\
\hline & & & & & & & Lower & Upper & & \\
\hline $\begin{array}{l}\text { Tenure with } \\
\text { organization }\end{array}$ & 28 & 14,422 & .02 & .02 & .06 & 38.15 & -.01 & .05 & $73.37^{*}$ & 507 \\
\hline $\begin{array}{c}\text { Tenure with } \\
\text { supervisor }\end{array}$ & 3 & 1,103 & -.03 & -.03 & .03 & 74.71 & -.09 & .03 & 4.02 & 2 \\
\hline Sex & 42 & 25,272 & .03 & .03 & .07 & 29.50 & .01 & .05 & $142.26^{*}$ & 1,326 \\
\hline Education & 22 & 7,541 & .01 & .02 & .12 & 19.77 & -.04 & .07 & $111.29^{*}$ & 420 \\
\hline $\begin{array}{l}\text { Self-esteem and } \\
\text { efficacy }\end{array}$ & 10 & 3,086 & .34 & .43 & .15 & 14.42 & .26 & .42 & $51.32 *$ & 1,357 \\
\hline Optimism & 5 & 1,504 & .28 & .34 & .01 & 96.98 & .24 & .33 & 5.11 & 204 \\
\hline Mindfulness & 5 & 1,299 & .28 & .33 & .00 & 100.00 & .23 & .33 & 1.67 & 161 \\
\hline Agreeableness & 7 & 1,834 & .36 & .45 & .06 & 52.06 & .31 & .42 & 11.66 & 582 \\
\hline Conscientiousness & 6 & 1,588 & .29 & .36 & .18 & 12.33 & .16 & .42 & $45.10^{*}$ & 279 \\
\hline Neuroticism & 3 & 879 & -.28 & -.32 & .07 & 47.76 & -.36 & -.19 & $6.23 *$ & 43 \\
\hline Extraversion & 3 & 879 & .34 & .41 & .05 & 63.49 & .28 & .40 & 4.66 & 76 \\
\hline Openness & 3 & 879 & .02 & .03 & .09 & 42.37 & -.08 & .12 & $7.08 *$ & 4 \\
\hline Proactive personality & 4 & 660 & .26 & .32 & .00 & 100.00 & .19 & .33 & 1.77 & 25 \\
\hline Causality orientation & 5 & 1,132 & .15 & .22 & .10 & 42.94 & .06 & .24 & $10.53 *$ & 42 \\
\hline Extrinsic values & 8 & 3,950 & -.00 & -.00 & .07 & 36.49 & -.05 & .05 & $21.92 *$ & 28 \\
\hline Intrinsic values & 9 & 4,333 & .26 & .32 & .12 & 17.38 & .19 & .32 & $48.78^{*}$ & 918 \\
\hline
\end{tabular}

Note: $k=$ number of effect sizes; $N=$ total subject number; $r=$ mean sample-weighted correlation; $\rho=$ estimate of corrected correlation; $S D \rho=$ corrected standard deviation of corrected correlation; $\% S E=$ percentage of observed variance accounted for by sampling error; $95 \% \mathrm{CI}=95 \%$ confidence interval around the mean sample-weighted correlation; $Q=$ chi-square test for the homogeneity of true correlations across studies; Tolerance $=$ number of studies showing null results necessary to eliminate the observed overall effect. $* p<.05$.

The basic needs demonstrated significant relations with each of the individual difference variables (see Table 2), with the exception of openness to experience (to which only competence was significantly related) and extrinsic values (to which none of the needs related). The confidence intervals generally overlapped (indicating similar nomological networks for each need), although relatedness related less strongly to mindfulness compared to competence and more strongly to extraversion compared to autonomy.

As reported in Table 3, all three needs related negatively to role stressors, work-family conflict, and job insecurity, but the results for job demands were somewhat more mixed. Satisfaction of the needs for autonomy and competence related negatively to workload and emotional demands, yet while autonomy was unrelated to cognitive demands, competence was positively related. Satisfaction of the need for relatedness was also positively related to cognitive demands and unrelated to workload and emotional demands. The confidence intervals frequently overlapped, with the exception that autonomy related more strongly to role stressors and job insecurity than competence and relatedness and more strongly to role conflict and less strongly to cognitive demands than competence. The positive relations between cognitive demands and competence and relatedness may be unexpected but may be due to cognitive demands representing a form of challenge stressor (Crawford, Lepine, \& Rich, 2010). That is, cognitively demanding jobs may represent intellectual challenges for 
Table 3

Meta-Analytic Relationships Between Needs, Job Stressors, and Job Resources

\begin{tabular}{|c|c|c|c|c|c|c|c|c|c|c|}
\hline \multirow[b]{2}{*}{ Variables } & \multirow[b]{2}{*}{$k$} & \multirow[b]{2}{*}{$N$} & \multirow[b]{2}{*}{$r$} & \multirow[b]{2}{*}{$\rho$} & \multirow[b]{2}{*}{$S D \rho$} & \multirow[b]{2}{*}{$\% S E$} & \multicolumn{2}{|c|}{$95 \% \mathrm{CI}$} & \multirow[b]{2}{*}{$Q$} & \multirow[b]{2}{*}{ Tolerance } \\
\hline & & & & & & & Lower & Upper & & \\
\hline \multicolumn{11}{|l|}{ Autonomy } \\
\hline Job demands & 16 & 6,255 & -.13 & -.16 & .21 & 7.50 & -.22 & -.05 & $209.62 *$ & 905 \\
\hline Workload & 16 & 6,255 & -.16 & -.19 & .22 & 6.76 & -.25 & -.06 & $231.82 *$ & \\
\hline $\begin{array}{c}\text { Emotional } \\
\text { demands }\end{array}$ & 7 & 2,904 & -.12 & -.14 & .06 & 46.98 & -.17 & -.07 & $14.75^{*}$ & \\
\hline $\begin{array}{l}\text { Cognitive } \\
\text { demands }\end{array}$ & 6 & 2,207 & .04 & .04 & .06 & 54.82 & -.01 & .07 & 10.92 & \\
\hline Role stressors & 10 & 3,500 & -.44 & -.54 & .14 & 12.88 & -.51 & -.36 & $66.49^{*}$ & 2,086 \\
\hline Role ambiguity & 7 & 1,814 & -.35 & -.43 & .14 & 19.43 & -.44 & -.25 & $33.79^{*}$ & \\
\hline Role conflict & 7 & 2,561 & -.51 & -.64 & .05 & 40.30 & -.56 & -.47 & $14.18^{*}$ & \\
\hline $\begin{array}{l}\text { Work-family } \\
\text { conflict }\end{array}$ & 9 & 2,830 & -.19 & -.24 & .07 & 48.45 & -.25 & -.14 & $18.09^{*}$ & 360 \\
\hline Job insecurity & 3 & 3,943 & -.34 & -.41 & .00 & 100.00 & -.36 & -.31 & 1.29 & 396 \\
\hline $\begin{array}{l}\text { Organizational } \\
\text { politics }\end{array}$ & 4 & 837 & -.45 & -.59 & .03 & 67.57 & -.50 & -.39 & 4.61 & 239 \\
\hline Skill variety & 15 & 5,391 & .37 & .49 & .16 & 11.22 & .30 & .44 & $82.64 *$ & 3,298 \\
\hline Task identity & 3 & 996 & .37 & .46 & .00 & 100.00 & .31 & .42 & 2.15 & 139 \\
\hline $\begin{array}{l}\text { Task } \\
\text { significance }\end{array}$ & 3 & 996 & .28 & .35 & .00 & 100.00 & .22 & .34 & 1.51 & 82 \\
\hline Job autonomy & 18 & 12,060 & .38 & .48 & .17 & 5.68 & .32 & .45 & $160.69^{*}$ & 10,158 \\
\hline Social support & 15 & 5,307 & .32 & .41 & .12 & 18.45 & .26 & .38 & $53.16^{*}$ & 2,885 \\
\hline Feedback & 4 & 7,032 & .33 & .42 & .07 & 10.54 & .26 & .39 & $20.36^{*}$ & 880 \\
\hline \multicolumn{11}{|l|}{ Competence } \\
\hline Job demands & 16 & 6,251 & -.09 & -.11 & .16 & 12.28 & -.15 & -.02 & $129.41 *$ & 431 \\
\hline Workload & 16 & 6,251 & -.08 & -.10 & .18 & 9.81 & -.16 & -.00 & $162.40^{*}$ & \\
\hline $\begin{array}{c}\text { Emotional } \\
\text { demands }\end{array}$ & 7 & 2,904 & -.09 & -.12 & .05 & 61.83 & -.13 & -.06 & 11.27 & \\
\hline $\begin{array}{r}\text { Cognitive } \\
\text { demands }\end{array}$ & 6 & 2,207 & .13 & .16 & .00 & 100.00 & .09 & .17 & 3.85 & \\
\hline Role stressors & 10 & 3,500 & -.24 & -.30 & .07 & 46.71 & -.29 & -.20 & $20.79 *$ & 707 \\
\hline Role ambiguity & 7 & 1,814 & -.35 & -.43 & .00 & 100.00 & -.39 & -.31 & 2.82 & \\
\hline Role conflict & 7 & 2,561 & -.19 & -.24 & .00 & 100.00 & -.23 & -.15 & 3.22 & \\
\hline $\begin{array}{l}\text { Work-family } \\
\text { conflict }\end{array}$ & 9 & 2,827 & -.13 & -.16 & .05 & 65.21 & -.16 & -.09 & 13.60 & 144 \\
\hline Job insecurity & 3 & 3,943 & -.24 & -.28 & .00 & 100.00 & -.27 & -.21 & 1.94 & 210 \\
\hline $\begin{array}{l}\text { Organizational } \\
\text { politics }\end{array}$ & 4 & 837 & -.37 & -.49 & .02 & 66.15 & -.43 & -.31 & 4.25 & 154 \\
\hline Skill variety & 15 & 5,386 & .19 & .25 & .08 & 39.17 & .15 & .23 & $34.02 *$ & 998 \\
\hline Task identity & 3 & 996 & .33 & .42 & .08 & 34.68 & .24 & .43 & $7.53^{*}$ & 115 \\
\hline $\begin{array}{l}\text { Task } \\
\text { significance }\end{array}$ & 3 & 996 & .33 & .41 & .05 & 60.80 & .27 & .38 & 4.72 & 118 \\
\hline Job autonomy & 18 & 12,058 & .15 & .19 & .11 & 14.38 & .10 & .20 & $121.35^{*}$ & 2,001 \\
\hline Social support & 15 & 5,303 & .13 & .17 & .13 & 21.46 & .08 & .19 & $67.34 *$ & 573 \\
\hline Feedback & 4 & 7,032 & .11 & .14 & .14 & 4.19 & .00 & .22 & $89.98 *$ & 230 \\
\hline
\end{tabular}


Table 3 (continued)

\begin{tabular}{|c|c|c|c|c|c|c|c|c|c|c|}
\hline \multirow[b]{2}{*}{ Variables } & \multirow[b]{2}{*}{$k$} & \multirow[b]{2}{*}{$N$} & \multirow[b]{2}{*}{$r$} & \multirow[b]{2}{*}{$\rho$} & \multirow[b]{2}{*}{$S D \rho$} & \multirow[b]{2}{*}{$\% S E$} & \multicolumn{2}{|c|}{$95 \% \mathrm{CI}$} & \multirow[b]{2}{*}{$Q$} & \multirow[b]{2}{*}{ Tolerance } \\
\hline & & & & & & & Lower & Upper & & \\
\hline \multicolumn{11}{|l|}{ Relatedness } \\
\hline Job demands & 16 & 6,255 & -.06 & -.07 & .19 & 8.99 & -.14 & .02 & $177.42 *$ & 574 \\
\hline Workload & 16 & 6,255 & -.07 & -.09 & .19 & 9.03 & -.15 & .01 & $176.57^{*}$ & \\
\hline $\begin{array}{c}\text { Emotional } \\
\text { demands }\end{array}$ & 7 & 2,904 & -.01 & -.01 & .06 & 50.66 & -.06 & .04 & $13.82 *$ & \\
\hline $\begin{array}{r}\text { Cognitive } \\
\text { demands }\end{array}$ & 6 & 2,207 & .09 & .11 & .00 & 100.00 & .05 & .13 & 4.46 & \\
\hline Role stressors & 10 & 3,500 & -.30 & -.37 & .01 & 90.16 & -.33 & -.27 & 10.40 & 906 \\
\hline $\begin{array}{l}\text { Work-family } \\
\text { conflict }\end{array}$ & 9 & 2,830 & -.14 & -.17 & .00 & 100.00 & -.17 & -.10 & 4.01 & 131 \\
\hline Job insecurity & 3 & 3,943 & -.23 & -.27 & .00 & 100.00 & -.26 & -.20 & 0.86 & 182 \\
\hline $\begin{array}{l}\text { Organizational } \\
\text { politics }\end{array}$ & 4 & 837 & -.35 & -.42 & .09 & 38.40 & -.45 & -.26 & $9.88^{*}$ & 149 \\
\hline Skill variety & 15 & 5,390 & .24 & .32 & .02 & 73.94 & .22 & .27 & 16.68 & 1,436 \\
\hline Task identity & 3 & 996 & .30 & .38 & .06 & 52.25 & .24 & .35 & 5.25 & 88 \\
\hline $\begin{array}{l}\text { Task } \\
\text { significance }\end{array}$ & 3 & 996 & .24 & .31 & .01 & 98.30 & .18 & .30 & 3.02 & 58 \\
\hline Job autonomy & 18 & 12,060 & .23 & .28 & .10 & 16.56 & .19 & .27 & $100.53 *$ & 2,211 \\
\hline Social support & 15 & 5,307 & .44 & .54 & .08 & 29.25 & .40 & .48 & $39.78 *$ & 5,110 \\
\hline Feedback & 4 & 7,032 & .28 & .35 & .04 & 31.38 & .24 & .32 & $9.24 *$ & 531 \\
\hline
\end{tabular}

Note: $k=$ number of effect sizes; $N=$ total subject number; $r=$ mean sample-weighted correlation; $\rho=$ estimate of corrected correlation; $S D \rho=$ corrected standard deviation of corrected correlation; $\% S E=$ percentage of observed variance accounted for by sampling error; $95 \% \mathrm{CI}=95 \%$ confidence interval around the mean sample-weighted correlation; $Q=$ chi-square test for the homogeneity of true correlations across studies; Tolerance = number of studies showing null results necessary to eliminate the observed overall effect.

$* p<.05$.

employees, increasing their sense of competence. The reason for a positive relation with relatedness is less clear, but cognitively demanding jobs may also be more likely to require teams to address the demands, leading to an increase in relatedness.

In general, the basic needs demonstrated positive and significant relations with all job resources (see Table 3). A number of confidence intervals did not overlap: The need for autonomy had the strongest relation with job autonomy, while the need for relatedness was most strongly related to social support, as might be expected. The confidence intervals for the relation of competence with both types of social support also did not overlap with the confidence intervals of the other two needs, and the confidence interval for the relation of autonomy with coworker support did not overlap with the confidence interval for relatedness.

As reported in Table 4, the basic needs generally demonstrated positive relations with the leader and organizational variables, the different fairness perceptions, and person-environment fit and negative relations with mistreatment. The confidence intervals mostly overlapped, except that - compared to the relation with competence - perceived organizational support and person-environment fit related more strongly to autonomy. This may suggest that satisfaction of the need for competence is more related to one's task than to the organizational context. 


\section{Table 4}

\section{Meta-Analytic Relationships Between Needs and Organizational Context}

\begin{tabular}{|c|c|c|c|c|c|c|c|c|c|c|}
\hline \multirow[b]{2}{*}{ Variables } & \multirow[b]{2}{*}{$k$} & \multirow[b]{2}{*}{$N$} & \multirow[b]{2}{*}{$r$} & \multirow[b]{2}{*}{$\rho$} & \multirow[b]{2}{*}{$S D \rho$} & \multirow[b]{2}{*}{$\% S E$} & \multicolumn{2}{|c|}{$95 \% \mathrm{CI}$} & \multirow[b]{2}{*}{$Q$} & \multirow[b]{2}{*}{ Tolerance } \\
\hline & & & & & & & Lower & Upper & & \\
\hline \multicolumn{11}{|l|}{ Autonomy } \\
\hline $\begin{array}{l}\text { Leader autonomy } \\
\text { support }\end{array}$ & 13 & 4,642 & .51 & .65 & .18 & 6.78 & .42 & .59 & $96.83 *$ & 4,963 \\
\hline $\begin{array}{l}\text { Leader relatedness } \\
\text { support }\end{array}$ & 3 & 1,303 & .52 & .63 & .00 & 100.00 & .48 & .56 & 1.65 & 390 \\
\hline $\begin{array}{l}\text { Perceived } \\
\text { organizational } \\
\text { support }\end{array}$ & 11 & 3,995 & .51 & .63 & .11 & 14.03 & .45 & .58 & $40.50^{*}$ & 4,207 \\
\hline $\begin{array}{l}\text { Organizational } \\
\text { exchange }\end{array}$ & 8 & 1,806 & .29 & .38 & .49 & 2.71 & .03 & .54 & $264.52 *$ & 843 \\
\hline $\begin{array}{l}\text { Positive leader } \\
\text { behavior }\end{array}$ & 7 & 5,482 & .36 & .42 & .20 & 3.18 & .23 & .49 & $134.58 *$ & 1,992 \\
\hline Fairness perceptions & 14 & 4,022 & .33 & .41 & .13 & 18.26 & .26 & .39 & $48.85^{*}$ & 2,269 \\
\hline Distributive justice & 5 & 1,467 & .25 & .30 & .15 & 15.77 & .13 & .37 & $28.66^{*}$ & \\
\hline Procedural justice & 11 & 3,086 & .35 & .43 & .15 & 13.89 & .27 & .43 & $48.65^{*}$ & \\
\hline Interactional justice & 6 & 1,833 & .33 & .44 & .17 & 12.41 & .21 & .44 & $24.84 *$ & \\
\hline $\begin{array}{l}\text { Person-environment } \\
\text { fit }\end{array}$ & 6 & 2,834 & .46 & .57 & .09 & 17.27 & .39 & .53 & $26.00^{*}$ & 1,060 \\
\hline $\begin{array}{l}\text { Leader-member } \\
\text { exchange }\end{array}$ & 6 & 1,816 & .63 & .74 & .13 & 8.34 & .53 & .73 & $41.79^{*}$ & 1,430 \\
\hline Mistreatment & 7 & 3,059 & -.45 & -.59 & .22 & 4.38 & -.59 & -.32 & $40.13^{*}$ & 1,137 \\
\hline \multicolumn{11}{|l|}{ Competence } \\
\hline $\begin{array}{l}\text { Leader autonomy } \\
\text { support }\end{array}$ & 13 & 4,642 & .30 & .38 & .11 & 22.41 & .24 & .35 & $49.20^{*}$ & 1,802 \\
\hline $\begin{array}{l}\text { Leader relatedness } \\
\text { support }\end{array}$ & 3 & 1,303 & .38 & .46 & .12 & 12.87 & .25 & .51 & $14.66^{*}$ & 213 \\
\hline $\begin{array}{l}\text { Perceived } \\
\text { organizational } \\
\text { support }\end{array}$ & 11 & 3,995 & .34 & .42 & .07 & 39.89 & .30 & .38 & $23.44^{*}$ & 1,838 \\
\hline $\begin{array}{l}\text { Organizational } \\
\text { exchange }\end{array}$ & 8 & 1,806 & .30 & .40 & .37 & 4.47 & .10 & .50 & $149.03 *$ & 760 \\
\hline $\begin{array}{l}\text { Positive leader } \\
\text { behavior }\end{array}$ & 7 & 5,482 & .34 & .40 & .12 & 7.81 & .26 & .43 & $57.42 *$ & 1,412 \\
\hline Fairness perceptions & 14 & 4,022 & .27 & .32 & .10 & 27.51 & .21 & .32 & $42.23 *$ & 1,444 \\
\hline Distributive justice & 5 & 1,467 & .13 & .15 & .11 & 26.45 & .03 & .23 & $18.61 *$ & \\
\hline Procedural justice & 11 & 3,086 & .26 & .31 & .12 & 23.44 & .19 & .33 & $40.85 *$ & \\
\hline Interactional justice & 6 & 1,833 & .35 & .46 & .02 & 62.90 & .31 & .39 & 6.47 & \\
\hline $\begin{array}{l}\text { Person-environment } \\
\text { fit }\end{array}$ & 6 & 2,829 & .16 & .20 & .05 & 53.90 & .11 & .21 & 10.86 & 174 \\
\hline $\begin{array}{l}\text { Leader-member } \\
\text { exchange }\end{array}$ & 6 & 1,816 & .53 & .63 & .21 & 4.89 & .38 & .68 & $86.73^{*}$ & 925 \\
\hline Mistreatment & 7 & 3,059 & -.30 & -.38 & .09 & 22.08 & -.37 & -.23 & $19.77^{*}$ & 651 \\
\hline \multicolumn{11}{|l|}{ Relatedness } \\
\hline $\begin{array}{l}\text { Leader autonomy } \\
\text { support }\end{array}$ & 14 & 5,051 & .32 & .39 & .08 & 31.91 & .27 & .36 & $35.44 *$ & 2,481 \\
\hline
\end{tabular}


Table 4 (continued)

\begin{tabular}{|c|c|c|c|c|c|c|c|c|c|c|}
\hline \multirow[b]{2}{*}{ Variables } & \multirow[b]{2}{*}{$k$} & \multirow[b]{2}{*}{$N$} & \multirow[b]{2}{*}{$r$} & \multirow[b]{2}{*}{$\rho$} & \multirow[b]{2}{*}{$S D \rho$} & \multirow[b]{2}{*}{$\% S E$} & \multicolumn{2}{|c|}{$95 \% \mathrm{CI}$} & \multirow[b]{2}{*}{$Q$} & \multirow[b]{2}{*}{ Tolerance } \\
\hline & & & & & & & Lower & Upper & & \\
\hline $\begin{array}{l}\text { Leader relatedness } \\
\text { support }\end{array}$ & 3 & 1,303 & .52 & .59 & .08 & 17.98 & .42 & .61 & $9.24^{*}$ & 386 \\
\hline $\begin{array}{l}\text { Perceived } \\
\text { organizational } \\
\text { support }\end{array}$ & 11 & 3,995 & .44 & .52 & .08 & 24.74 & .39 & .49 & $9.24^{*}$ & 3,017 \\
\hline $\begin{array}{l}\text { Organizational } \\
\text { exchange }\end{array}$ & 8 & 1,806 & .29 & .35 & .32 & 4.87 & .10 & .48 & $9.24 *$ & 749 \\
\hline $\begin{array}{l}\text { Positive leader } \\
\text { behavior }\end{array}$ & 7 & 5,482 & .36 & .40 & .24 & 2.09 & .19 & .52 & $9.24 *$ & 2,078 \\
\hline Fairness perceptions & 14 & 4,022 & .35 & .41 & .10 & 25.94 & .30 & .41 & $9.24 *$ & 2,546 \\
\hline Distributive justice & 5 & 1,467 & .27 & .31 & .09 & 32.35 & .18 & .35 & $14.38^{*}$ & \\
\hline Procedural justice & 11 & 3,086 & .40 & .47 & .12 & 19.04 & .33 & .47 & $51.34 *$ & \\
\hline Interactional justice & 6 & 1,833 & .36 & .43 & .14 & 15.40 & .26 & .46 & $35.58^{*}$ & \\
\hline $\begin{array}{l}\text { Person-environment } \\
\text { fit }\end{array}$ & 6 & 2,833 & .38 & .46 & .00 & 100.00 & .35 & .41 & 3.58 & 761 \\
\hline $\begin{array}{l}\text { Leader-member } \\
\text { exchange }\end{array}$ & 6 & 1,816 & .59 & .67 & .24 & 3.12 & .42 & .76 & $166.20^{*}$ & 1,162 \\
\hline Mistreatment & 7 & 3,059 & -.39 & -.46 & .11 & 15.37 & -.47 & -.32 & $39.28 *$ & 999 \\
\hline
\end{tabular}

Note: $k=$ number of effect sizes; $N=$ total subject number; $r=$ mean sample-weighted correlation; $\rho=$ estimate of corrected correlation; $S D \rho=$ corrected standard deviation of corrected correlation; $\% S E=$ percentage of observed variance accounted for by sampling error; $95 \% \mathrm{CI}=95 \%$ confidence interval around the mean sample-weighted correlation; $Q=$ chi-square test for the homogeneity of true correlations across studies; Tolerance = number of studies showing null results necessary to eliminate the observed overall effect.

$* p<.05$.

\section{Relations Between Needs and Outcome Variables}

Reported in Tables 5 and 6 are meta-analytic relations between the basic needs and indicators of well-being, job attitudes, job behaviors, and motivation. As we discuss the relative effects of the needs on these outcomes in our subsequent section on the RW analyses, in this section, we will simply summarize the patterns of the mean sample-weighted correlations.

For well-being, each of the basic needs demonstrated significant relations with the indicators of well-being. For job attitudes, each of the basic needs was positively related to job satisfaction and affective commitment and negatively related to turnover intentions. For job behaviors, each of the basic needs had positive relations with the performance measures and effort while being negatively related to deviance; however, only the needs for autonomy and relatedness related significantly and negatively to absenteeism while competence was unrelated. Finally, for motivation, each of the basic needs was negatively related to amotivation. The satisfaction of the need for autonomy and competence demonstrated negative and significant relations with external motivation, whereas the need for relatedness was unrelated to external motivation. Each basic need had positive significant relations with introjected, identified, and intrinsic motivation. 
Table 5

Meta-Analytic Relationships Between Needs, Well-Being, and Job Attitudes

\begin{tabular}{|c|c|c|c|c|c|c|c|c|c|c|}
\hline \multirow[b]{2}{*}{ Variables } & \multirow[b]{2}{*}{$k$} & \multirow[b]{2}{*}{$N$} & \multirow[b]{2}{*}{$r$} & \multirow[b]{2}{*}{$\rho$} & \multirow[b]{2}{*}{$S D \rho$} & \multirow[b]{2}{*}{$\% S E$} & \multicolumn{2}{|c|}{$95 \% \mathrm{CI}$} & \multirow[b]{2}{*}{$Q$} & \multirow[b]{2}{*}{ Tolerance } \\
\hline & & & & & & & Lower & Upper & & \\
\hline \multicolumn{11}{|l|}{ Autonomy } \\
\hline Positive affect & 11 & 2,811 & .49 & .60 & .06 & 41.69 & .45 & .54 & $19.87^{*}$ & 2,157 \\
\hline Engagement & 50 & 25,562 & .54 & .65 & .06 & 18.82 & .52 & .56 & $109.34 *$ & 93,322 \\
\hline General well-being & 16 & 5,602 & .44 & .52 & .06 & 36.57 & .40 & .47 & $34.59 *$ & 6,104 \\
\hline Life satisfaction & 7 & 3,182 & .23 & .31 & .09 & 30.21 & .17 & .29 & $18.43^{*}$ & 440 \\
\hline Negative affect & 9 & 2,019 & -.37 & -.46 & .04 & 65.16 & -.41 & -.33 & 11.86 & 743 \\
\hline Strain & 24 & 7,278 & -.34 & -.42 & .10 & 24.71 & -.38 & -.30 & $78.27^{*}$ & 6,815 \\
\hline Burnout & 45 & 19,203 & -.50 & -.60 & .12 & 10.41 & -.53 & -.47 & $192.60 *$ & 62,774 \\
\hline Job satisfaction & 34 & 12,519 & .54 & .69 & .13 & 8.53 & .50 & .59 & $103.61 *$ & 40,377 \\
\hline $\begin{array}{l}\text { Affective } \\
\text { commitment }\end{array}$ & 28 & 16,984 & .48 & .62 & .11 & 10.36 & .45 & .52 & $116.48^{*}$ & 30,368 \\
\hline $\begin{array}{l}\text { Turnover } \\
\text { intentions }\end{array}$ & 26 & 14,448 & -.31 & -.38 & .30 & 2.58 & -.40 & -.21 & $842.71^{*}$ & 5,627 \\
\hline \multicolumn{11}{|l|}{ Competence } \\
\hline Positive affect & 11 & 2,811 & .48 & .59 & .06 & 49.47 & .44 & .52 & $19.57 *$ & 2,052 \\
\hline Engagement & 50 & 25,562 & .33 & .38 & .12 & 13.23 & .30 & .36 & $299.96^{*}$ & 38,965 \\
\hline General well-being & 16 & 5,602 & .49 & .58 & .07 & 25.44 & .45 & .53 & $38.05^{*}$ & 7,702 \\
\hline Life satisfaction & 7 & 3,182 & .25 & .32 & .11 & 21.33 & .18 & .32 & 28.94 & 457 \\
\hline Negative affect & 9 & 2,019 & -.32 & -.40 & .07 & 48.75 & -.37 & -.26 & $16.93 *$ & 559 \\
\hline Strain & 24 & 7,278 & -.31 & -.38 & .15 & 14.82 & -.37 & -.26 & $131.68^{*}$ & 5,807 \\
\hline Burnout & 45 & 19,203 & -.25 & -.30 & .16 & 10.12 & -.29 & -.21 & $383.37 *$ & 17,312 \\
\hline Job satisfaction & 34 & 12,515 & .40 & .50 & .17 & 9.30 & .35 & .44 & $209.09 *$ & 20,788 \\
\hline $\begin{array}{l}\text { Affective } \\
\text { commitment }\end{array}$ & 28 & 16,984 & .21 & .27 & .14 & 13.33 & .17 & .26 & $236.86^{*}$ & 7,390 \\
\hline $\begin{array}{l}\text { Turnover } \\
\text { intentions }\end{array}$ & 26 & 14,448 & -.05 & -.07 & .17 & 8.81 & -.11 & -.00 & $292.57^{*}$ & 1,657 \\
\hline \multicolumn{11}{|l|}{ Relatedness } \\
\hline Positive affect & 11 & 2,811 & .41 & .48 & .09 & 29.91 & .35 & .47 & $33.70^{*}$ & 1,470 \\
\hline Engagement & 51 & 25,971 & .40 & .48 & .08 & 21.94 & .37 & .42 & $151.28 *$ & 53,981 \\
\hline General well-being & 16 & 5,602 & .37 & .44 & .13 & 14.24 & .31 & .43 & $96.98^{*}$ & 4,470 \\
\hline Life satisfaction & 7 & 3,182 & .26 & .33 & .05 & 55.84 & .23 & .30 & 11.67 & 505 \\
\hline Negative affect & 9 & 2,019 & -.27 & -.33 & .02 & 88.66 & -.31 & -.23 & 9.53 & 417 \\
\hline Strain & 23 & 7,155 & -.30 & -.36 & .14 & 15.57 & -.36 & -.25 & $125.94 *$ & 5,177 \\
\hline Burnout & 46 & 19,612 & -.32 & -.39 & .11 & 18.00 & -.35 & -.29 & $213.19 *$ & 27,735 \\
\hline Job satisfaction & 34 & 12,519 & .42 & .52 & .09 & 21.87 & .39 & .45 & $95.30 *$ & 25,360 \\
\hline $\begin{array}{l}\text { Affective } \\
\text { commitment }\end{array}$ & 28 & 16,984 & .47 & .60 & .11 & 10.36 & .43 & .50 & $136.02 *$ & 25,533 \\
\hline $\begin{array}{l}\text { Turnover } \\
\text { intentions }\end{array}$ & 26 & 14,448 & -.21 & -.28 & .23 & 4.84 & -.29 & -.15 & $477.81^{*}$ & 3,096 \\
\hline
\end{tabular}

Note: $k=$ number of effect sizes; $N=$ total subject number; $r=$ mean sample-weighted correlation; $\rho=$ estimate of corrected correlation; $S D \rho=$ corrected standard deviation of corrected correlation; $\% S E=$ percentage of observed variance accounted for by sampling error; $95 \% \mathrm{CI}=95 \%$ confidence interval around the mean sample-weighted correlation; $Q=$ chi-square test for the homogeneity of true correlations across studies; Tolerance $=$ number of studies showing null results necessary to eliminate the observed overall effect.

$* p<.05$. 
Table 6

Meta-Analytic Relationships Between Needs, Job Behaviors, and Motivation

\begin{tabular}{|c|c|c|c|c|c|c|c|c|c|c|}
\hline \multirow[b]{2}{*}{ Variables } & \multirow[b]{2}{*}{$k$} & \multirow[b]{2}{*}{$N$} & \multirow[b]{2}{*}{$r$} & \multirow[b]{2}{*}{$\rho$} & \multirow[b]{2}{*}{$S D \rho$} & \multirow[b]{2}{*}{$\% S E$} & \multicolumn{2}{|c|}{$95 \% \mathrm{CI}$} & \multirow[b]{2}{*}{$Q$} & \multirow[b]{2}{*}{ Tolerance } \\
\hline & & & & & & & Lower & Upper & & \\
\hline \multicolumn{11}{|l|}{ Autonomy } \\
\hline Task performance & 21 & 5,261 & .23 & .29 & .10 & 36.78 & .19 & .27 & $50.78^{*}$ & 191 \\
\hline Creative performance & 4 & 840 & .31 & .37 & .17 & 16.15 & .15 & .46 & $22.42^{*}$ & 111 \\
\hline Proactive performance & 23 & 5,581 & .27 & .35 & .12 & 28.05 & .22 & .32 & $64.81^{*}$ & 2,968 \\
\hline Job crafting & 4 & 778 & .29 & .35 & .14 & 25.16 & .16 & .42 & $15.39^{*}$ & \\
\hline OCB individual & 8 & 1,880 & .22 & .29 & .07 & 54.18 & .16 & .28 & 12.59 & \\
\hline OCB organization & 8 & 1,880 & .27 & .35 & .05 & 62.83 & .22 & .31 & 10.01 & \\
\hline Effort & 3 & 1,177 & .21 & .25 & .02 & 83.46 & .15 & .26 & 3.39 & 33 \\
\hline Deviance behavior & 9 & 3,330 & -.19 & -.25 & .03 & 70.03 & -.22 & -.16 & 10.16 & 338 \\
\hline Absenteeism & 16 & 6,793 & -.10 & -.11 & .03 & 73.27 & -.12 & -.08 & 21.80 & 363 \\
\hline Amotivation & 5 & 3,030 & -.29 & -.34 & .02 & 74.41 & -.33 & -.26 & 6.52 & 446 \\
\hline External motivation & 31 & 12,522 & -.07 & -.09 & .12 & 22.20 & -.11 & -.04 & $138.47 *$ & 1,544 \\
\hline Introjected motivation & 27 & 10,712 & .05 & .06 & .10 & 25.73 & .01 & .09 & $104.63 *$ & 838 \\
\hline Identified motivation & 31 & 11,970 & .32 & .40 & .15 & 12.61 & .27 & .37 & $207.21 *$ & 12,995 \\
\hline Intrinsic motivation & 34 & 12,594 & .54 & .64 & .11 & 13.20 & .51 & .58 & $182.43^{*}$ & 39,319 \\
\hline \multicolumn{11}{|l|}{ Competence } \\
\hline Task performance & 21 & 5,261 & .33 & .40 & .14 & 18.94 & .27 & .38 & $98.03 *$ & 3,326 \\
\hline Creative performance & 4 & 840 & .29 & .34 & .17 & 16.41 & .14 & .44 & $22.60^{*}$ & 100 \\
\hline Proactive performance & 23 & 5,581 & .30 & .37 & .10 & 33.25 & .26 & .34 & $57.00^{*}$ & 3,555 \\
\hline Job crafting & 4 & 778 & .27 & .33 & .20 & 13.56 & .10 & .45 & $28.58^{*}$ & \\
\hline OCB individual & 8 & 1,880 & .26 & .32 & .00 & 100.00 & .21 & .29 & 6.85 & \\
\hline OCB organization & 8 & 1,880 & .28 & .36 & .04 & 68.99 & .24 & .32 & 10.40 & \\
\hline Effort & 3 & 1,173 & .30 & .35 & .10 & 20.98 & .19 & .42 & $13.37 *$ & 70 \\
\hline Deviance behavior & 9 & 3,330 & -.18 & -.23 & .13 & 18.93 & -.26 & -.10 & $41.50^{*}$ & 370 \\
\hline Absenteeism & 16 & 6,793 & .01 & .01 & .06 & 40.31 & -.03 & .05 & $39.69^{*}$ & 156 \\
\hline Amotivation & 5 & 3,030 & -.20 & -.24 & .11 & 15.35 & -.29 & -.12 & $32.15^{*}$ & 238 \\
\hline External motivation & 31 & 12,522 & -.05 & -.06 & .12 & 19.85 & -.09 & -.01 & $155.53 *$ & 1,181 \\
\hline Introjected motivation & 27 & 10,712 & .05 & .07 & .14 & 14.66 & .01 & .10 & $183.62 *$ & 1,120 \\
\hline Identified motivation & 31 & 11,970 & .25 & .31 & .09 & 28.54 & .22 & .28 & $95.98 *$ & 8,210 \\
\hline Intrinsic motivation & 34 & 12,594 & .28 & .32 & .08 & 32.02 & .25 & .31 & $100.22 *$ & 12,059 \\
\hline \multicolumn{11}{|l|}{ Relatedness } \\
\hline Task performance & 21 & 5,261 & .21 & .26 & .14 & 21.83 & .16 & .27 & $89.49^{*}$ & 1,641 \\
\hline Creative performance & 4 & 840 & .28 & .31 & .12 & 27.25 & .16 & .40 & $14.04 *$ & 89 \\
\hline Proactive performance & 23 & 5,581 & .30 & .36 & .10 & 33.53 & .26 & .34 & $59.21^{*}$ & 3,334 \\
\hline Job crafting & 4 & 778 & .26 & .32 & .12 & 30.74 & .14 & .38 & $12.61^{*}$ & \\
\hline OCB individual & 8 & 1,880 & .32 & .37 & .07 & 46.84 & .26 & .38 & $16.19^{*}$ & \\
\hline OCB organization & 8 & 1,880 & .29 & .33 & .09 & 38.76 & .22 & .35 & $19.53 *$ & \\
\hline Effort & 3 & 1,177 & .17 & .20 & .07 & 37.35 & .08 & .27 & $7.99 *$ & 28 \\
\hline Deviance behavior & 9 & 3,330 & -.16 & -.20 & .12 & 20.00 & -.24 & -.09 & $43.12 *$ & 315 \\
\hline Absenteeism & 16 & 6,793 & -.05 & -.06 & .03 & 80.05 & -.07 & -.02 & 19.96 & 124 \\
\hline Amotivation & 5 & 3,030 & -.22 & -.26 & .07 & 28.88 & -.29 & -.16 & $17.03 *$ & 274 \\
\hline External motivation & 31 & 12,522 & -.01 & -.02 & .11 & 23.49 & -.05 & .02 & $131.93 *$ & 806 \\
\hline Introjected motivation & 27 & 10,712 & .06 & .07 & .09 & 31.09 & .02 & .09 & $86.43^{*}$ & 677 \\
\hline Identified motivation & 31 & 11,970 & .24 & .30 & .08 & 35.38 & .21 & .27 & $79.06^{*}$ & 6,865 \\
\hline Intrinsic motivation & 34 & 12,594 & .36 & .44 & .06 & 44.77 & .34 & .38 & $64.24 *$ & 17,652 \\
\hline
\end{tabular}

Note: $k=$ number of effect sizes; $N=$ total subject number; $r=$ mean sample-weighted correlation; $\rho=$ estimate of corrected correlation; $S D \rho=$ corrected standard deviation of corrected correlation; $\% S E=$ percentage of observed variance accounted for by sampling error; $95 \% \mathrm{CI}=95 \%$ confidence interval around the mean sample-weighted correlation; $Q=$ chi-square test for the homogeneity of true correlations across studies; Tolerance $=$ number of studies showing null results necessary to eliminate the observed overall effect; $\mathrm{OCB}=$ organizational citizenship behavior.

$* p<.05$. 


\section{$R W$ Analyses of the Three Needs}

Table 7 provides the results of the RW analyses that test whether each need independently predicts measures of psychological growth, internalization, and well-being. Each need accounted for unique variance in intrinsic motivation, explaining $42 \%$ of the variance overall. With respect to predicting the different types of motivation involved in the internalization process, by and large, each need accounted for unique variation in amotivation, external motivation, introjected motivation, and identified motivation.

The only exceptions to this trend were that autonomy did not incrementally predict introjected motivation beyond competence and relatedness and that competence and relatedness were significantly positively related to introjected motivation (with relatedness also being positively related to external motivation). Although potentially surprising, such positive relations are perhaps not unexpected. Specifically, SDT argues that external and introjected motivation can occur when engaging in behaviors important to people we feel a sense of relatedness to (e.g., we provide sample exam questions because we know our students would like us if we did, not because we want to) or when engaging in behaviors we feel competent at (e.g., we may teach methodology because we are good at it and no one else in the department can teach it, not because we enjoy it; see Deci \& Ryan, 2000; Ryan \& Deci, 2000). However, we should also note the variance explained in external and introjected motivation was essentially negligible, with only $1 \%$ of the variance being accounted for by all three needs.

With respect to psychological well-being, each need accounted for unique variation in positive affect, general well-being, and life satisfaction, as well as in negative affect, strain, and burnout. The exception to this trend was in predicting engagement, where satisfaction of the need for competence did not predict incrementally beyond satisfaction of the needs for autonomy and relatedness. Across the various operationalizations of psychological well-being, the three needs accounted for between $15 \%$ and $46 \%$ of the variance in well-being outcomes.

With respect to job attitudes, each need accounted for unique variation in job satisfaction, affective commitment, and turnover intentions. However, satisfaction of the need for competence unexpectedly related negatively with affective commitment and positively with turnover intentions. These results contrast with SDT's prediction that need satisfaction should lead to more favorable outcomes (Gagné \& Deci, 2005). Notably, these results for competence emerged only in our RW analyses; the sample-weighted mean correlation between competence need satisfaction and affective commitment was significant and positive, while the sample-weighted mean correlation with turnover intentions was negative. Given the overlap between the three needs, we suspect that the variance associated with autonomy and relatedness needs to be accounted for before the counterintuitive effects of competence can be observed. Although this may represent a suppression effect, a speculative explanation may be that employees who feel competent see themselves as having the necessary knowledge, skills, and abilities that are valued by different employers and, hence, become less committed to their current employer and seek other opportunities elsewhere (Fugate, Kinicki, \& Ashforth, 2004).

Finally, with respect to predicting behavioral outcomes, the three basic needs each accounted for variance in effort, deviance behavior, absenteeism, and task, creative, and proactive performance; the only exception was that satisfaction of the need for relatedness did not incrementally predict effort and absenteeism. Notably, the relation between satisfaction of the need for competence and absenteeism was also positive. This unexpected relation 
Table 7

Relative Weight Analyses

\begin{tabular}{|c|c|c|c|c|c|c|c|c|c|c|c|c|}
\hline \multirow[b]{3}{*}{ Outcomes } & \multirow[b]{3}{*}{$N$} & \multicolumn{9}{|c|}{ Predictors } & \multirow[b]{3}{*}{$F$} & \multirow[b]{3}{*}{$R^{2}$} \\
\hline & & \multicolumn{3}{|c|}{ Autonomy } & \multicolumn{3}{|c|}{ Competence } & \multicolumn{3}{|c|}{ Relatedness } & & \\
\hline & & $\beta$ & RW & $\%$ & $\beta$ & $\mathrm{RW}$ & $\%$ & $\beta$ & $\mathrm{RW}$ & $\%$ & & \\
\hline Amotivation ${ }^{\mathrm{a}}$ & 5,167 & $-0.24^{*}$ & .07 & 52.9 & $-0.11 *$ & .03 & 22.9 & -0.09 & .03 & 24.2 & $264.58^{*}$ & .13 \\
\hline $\begin{array}{l}\text { External } \\
\text { motivation }^{\mathrm{a}}\end{array}$ & 14,613 & $-0.10^{*}$ & .01 & 70.4 & $-0.03 *$ & .00 & 21.8 & $0.05^{*}$ & .00 & 7.9 & $50.58^{*}$ & .01 \\
\hline $\begin{array}{l}\text { Introjected } \\
\text { motivation }^{\mathrm{a}}\end{array}$ & 13,302 & 0.01 & .00 & 19.2 & $0.05 *$ & .00 & 42.4 & $0.05^{*}$ & .00 & 38.4 & $34.11^{*}$ & .01 \\
\hline $\begin{array}{l}\text { Identified } \\
\text { motivation }^{\mathrm{a}}\end{array}$ & 11,756 & $0.28^{*}$ & .10 & 50.0 & $0.17 *$ & .05 & 28.3 & $0.09 *$ & .04 & 21.6 & $1,123.74 *$ & .19 \\
\hline $\begin{array}{l}\text { Intrinsic } \\
\text { motivation }^{\mathrm{a}}\end{array}$ & 14,662 & $0.55^{*}$ & .29 & 67.7 & $0.06^{*}$ & .04 & 10.4 & $0.11^{*}$ & .09 & 21.9 & $3,570.89 *$ & .42 \\
\hline Positive affect & 5,297 & $0.33^{*}$ & .18 & 38.3 & $0.35^{*}$ & .19 & 41.3 & $0.13 *$ & .09 & 20.4 & $1,506.54 *$ & .46 \\
\hline $\begin{array}{l}\text { General well- } \\
\text { being }\end{array}$ & 9,982 & $0.21^{*}$ & .12 & 30.2 & $0.40^{*}$ & .20 & 49.9 & $0.13^{*}$ & .08 & 19.9 & $2,216.57^{*}$ & .40 \\
\hline $\begin{array}{l}\text { Life } \\
\text { satisfaction }\end{array}$ & 5,950 & $0.09^{*}$ & .04 & 26.2 & $0.18^{*}$ & .05 & 35.8 & $0.20^{*}$ & .06 & 38.1 & $348.98^{*}$ & .15 \\
\hline Engagement & 32,907 & $0.57^{*}$ & .27 & 62.8 & -0.01 & .06 & 12.8 & $0.13^{*}$ & .11 & 24.5 & $4,915.63 *$ & .43 \\
\hline Negative affect & t 3,867 & $-0.32^{*}$ & .12 & 48.5 & $-0.20 *$ & .08 & 33.9 & $-0.05^{*}$ & .04 & 17.6 & $408.99 *$ & .24 \\
\hline Strain & 12,496 & $-0.23 *$ & .09 & 40.0 & $-0.19 *$ & .07 & 33.2 & $-0.14^{*}$ & .06 & 26.7 & $1,152.61 *$ & .22 \\
\hline Burnout $^{\mathrm{a}}$ & 18,397 & $-0.54^{*}$ & .26 & 70.1 & $-0.06^{*}$ & .04 & 10.8 & $-0.07 *$ & .07 & 19.2 & $3,556.09^{*}$ & .37 \\
\hline $\begin{array}{l}\text { Job } \\
\text { satisfaction }\end{array}$ & 19,656 & $0.53^{*}$ & .28 & 55.3 & $0.14^{*}$ & .11 & 21.5 & $0.14^{*}$ & .12 & 23.3 & $6,661.51^{*}$ & .50 \\
\hline $\begin{array}{l}\text { Affective } \\
\text { commitment }\end{array}$ & 24,771 & $0.49^{*}$ & .24 & 49.3 & $-0.18^{*}$ & .03 & 6.1 & $0.38^{*}$ & .22 & 44.5 & $7,757.66^{*}$ & .50 \\
\hline $\begin{array}{l}\text { Turnover } \\
\text { intentions }\end{array}$ & 21,960 & $-0.44^{*}$ & .12 & 65.6 & $0.24 *$ & .02 & 9.4 & $-0.12 *$ & .05 & 25.0 & $1,653.93 *$ & .18 \\
\hline $\begin{array}{l}\text { Task } \\
\text { performance }\end{array}$ & 9,437 & $0.05^{*}$ & .03 & 19.6 & $0.34 *$ & .11 & 64.1 & $0.08^{*}$ & .03 & 16.3 & $641.21^{*}$ & .17 \\
\hline $\begin{array}{l}\text { Creative } \\
\text { performance }\end{array}$ & 1,650 & $0.21 *$ & .07 & 40.2 & $0.18^{*}$ & .06 & 35.0 & $0.11^{*}$ & .04 & 24.8 & $111.13^{*}$ & .17 \\
\hline $\begin{array}{l}\text { Proactive } \\
\text { performance }\end{array}$ & 9,948 & $0.10^{*}$ & .05 & 26.5 & $0.22 *$ & .07 & 39.0 & $0.20^{*}$ & .07 & 34.5 & $774.16^{*}$ & .19 \\
\hline Effort & 2,292 & $0.06^{*}$ & .03 & 20.7 & 0.30 * & .09 & 67.7 & 0.03 & .02 & 11.6 & $110.65^{*}$ & .13 \\
\hline Deviance & 6,208 & $-0.15^{*}$ & .03 & 41.8 & $-0.12 *$ & .03 & 36.1 & $-0.06^{*}$ & .02 & 22.0 & $169.50^{*}$ & .08 \\
\hline Absenteeism & 11,829 & $-0.17^{*}$ & .01 & 67.3 & $0.11^{*}$ & .00 & 19.5 & -0.01 & .00 & 13.2 & $80.25^{*}$ & .02 \\
\hline
\end{tabular}

Note: $\mathrm{RW}=$ relative weight; $\%$ = rescaled relative weight (i.e., relative weight divided by full model $R^{2}$ ).

${ }^{a}$ Model estimated using corrected correlations between needs assessed by the Van den Broeck, Vansteenkiste, De Witte, Soenens, and Lens (2010) scale as a majority of the studies used the Van den Broeck et al. scale.

${ }^{*} p<.05$.

may be explained along similar lines as our explanation for the positive association between competence and turnover intentions, although we should also note that need satisfaction as a whole failed to meaningfully predict attendance as an outcome, accounting for only $2 \%$ of the variance. 


\section{Scale Used and Publication Status as Between-Study Moderators}

Table 8 summarizes the relations between needs and antecedent/outcome variables that differed depending on the scale used and publication status. With respect to the scale used, the individual needs correlated more strongly with each other when the Deci et al. (2001) scale was used versus when the Van den Broeck et al. (2010) measure was used. Outside of the correlations among the needs, there were a limited number of studies available to compare the findings for each scale. Nevertheless, the three needs generally demonstrated somewhat stronger relations with outcomes when the Deci et al. scale was used compared to when the Van den Broeck et al. scale was used, particularly for burnout, turnover intentions, and proactive performance. A reviewer also suggested we calculate the average reliability (assessed via Cronbach's alpha) for each of the three needs for both the Deci et al. and the Van den Broeck et al. scale. The Deci et al. measures for competence $(\alpha=.82)$ and relatedness $(\alpha=.82)$ were above the recommended .70 threshold (Nunnally \& Bernstein, 1994), but the measure for autonomy was not $(\alpha=.68)$. For the Van den Broeck et al. scale, the measures for autonomy $(\alpha=.79)$, competence $(\alpha=.83)$, and relatedness $(\alpha=.76)$ all surpassed the recommended threshold.

Finally, we examined publication status as a between-study moderator in our analyses. No clear trend emerged: While in some cases published studies yielded stronger effects, in other cases unpublished studies yielded stronger effects, and in still other cases the effects were comparable. Overall, then, there was no consistent or discernable trend with regard to how publication status affected the reported correlations. As such, our results did not provide evidence of publication bias (Rosenthal, 1979).

\section{Summary}

Taken together, the results of our meta-analysis are largely supportive for SDT. Below, we evaluate the three specific aims of our meta-analysis - whether the three needs incrementally predict measures of psychological growth, internalization, and well-being; whether averaging the three needs into an overall need satisfaction measure is appropriate; and whether the effects of need satisfaction varied across the measure used. Subsequently, we outline general recommendations for future research on basic psychological needs.

\section{Do Basic Psychological Needs Incrementally Predict Psychological Growth, Internalization, and Well-Being?}

The very definition of basic psychological needs in SDT requires that each need incrementally predict psychological growth, internalization, and well-being. By and large, our results support this proposition. In particular, intrinsic motivation and various indices of well-being were all predicted uniquely by satisfaction of each of the needs (with one exception regarding the relation of competence to engagement). From an SDT point of view, perhaps most interesting is that satisfaction of the need for relatedness was fairly strongly related to intrinsic motivation, given that past work had generally argued it plays a more distal role than autonomy or competence (Deci \& Ryan, 2000).

With respect to psychological well-being, one aspect is of particular note: In line with the criticism that SDT best explains positive but not negative or "dark sides" of human life 
Table 8

Publication Status and Scale Used as Between-Study Moderators

\begin{tabular}{|c|c|c|c|c|c|c|}
\hline \multirow[b]{2}{*}{ Effect } & \multicolumn{3}{|c|}{ Publication Status } & \multicolumn{3}{|c|}{ Scale Used } \\
\hline & $\rho_{\text {published }}$ & $\rho_{\text {unpublished }}$ & $Z$ & $\begin{array}{c}\rho_{\text {Deci, Ryan, Gagne, }} \\
\text { Leone, Usunov, \& } \\
\text { Kornazheva (2001) }\end{array}$ & $\begin{array}{c}\rho_{\text {Van den Broeck, }} \\
\text { Vansteenkiste, De Witte, } \\
\text { Soenens, \& Lens (2010) }\end{array}$ & $Z$ \\
\hline Autonomy - Competence & .59 & .52 & $7.40^{*}$ & .64 & .41 & $28.95^{*}$ \\
\hline Competence - Relatedness & .47 & .41 & $7.46^{*}$ & .50 & .30 & $21.47^{*}$ \\
\hline Autonomy - Relatedness & .61 & .61 & 0.00 & .65 & .56 & $12.76^{*}$ \\
\hline Autonomy - Age & .03 & .04 & -0.6 & .02 & .02 & 0.00 \\
\hline $\begin{array}{l}\text { Autonomy - Tenure with } \\
\text { organization }\end{array}$ & .06 & .03 & 1.60 & .04 & .05 & -0.50 \\
\hline Autonomy - Sex & .00 & .02 & -1.28 & .01 & -.01 & 1.42 \\
\hline Autonomy - Education & .12 & .09 & 1.29 & .05 & .09 & -1.44 \\
\hline Competence - Age & .05 & .09 & $-2.41 *$ & .09 & .00 & $6.07 *$ \\
\hline $\begin{array}{l}\text { Competence }- \text { Tenure } \\
\text { with organization }\end{array}$ & .09 & .09 & 0.00 & .09 & .12 & -1.60 \\
\hline Competence - Sex & -.02 & .01 & -1.92 & -.02 & -.02 & 0.00 \\
\hline Competence - Education & .06 & .00 & $2.55^{*}$ & .03 & .00 & 1.11 \\
\hline Relatedness - Age & -.02 & -.04 & 1.19 & -.06 & .01 & $-4.71^{*}$ \\
\hline $\begin{array}{l}\text { Relatedness - Tenure with } \\
\text { organization }\end{array}$ & .04 & .02 & 1.06 & .02 & .04 & -1.05 \\
\hline Relatedness - Sex & .02 & .05 & -1.93 & .05 & -.01 & $4.34^{*}$ \\
\hline Relatedness - Education & .05 & -.00 & $2.12 *$ & -.05 & .02 & $-2.58^{*}$ \\
\hline $\begin{array}{l}\text { Autonomy - Self-esteem/ } \\
\text { efficacy }\end{array}$ & .33 & .57 & $-8.54^{*}$ & - & - & - \\
\hline $\begin{array}{l}\text { Competence - Self- } \\
\text { esteem/efficacy }\end{array}$ & .46 & .62 & $-6.38^{*}$ & - & - & - \\
\hline $\begin{array}{l}\text { Competence - Extrinsic } \\
\text { values }\end{array}$ & -.02 & .11 & $-4.02 *$ & - & 一 & - \\
\hline $\begin{array}{l}\text { Competence - Intrinsic } \\
\text { values }\end{array}$ & .18 & .22 & -1.22 & - & - & - \\
\hline $\begin{array}{l}\text { Relatedness - Self-esteem/ } \\
\text { efficacy }\end{array}$ & .32 & .55 & $-7.91 *$ & - & - & - \\
\hline $\begin{array}{l}\text { Relatedness - Extrinsic } \\
\text { values }\end{array}$ & -.01 & .00 & -0.31 & - & - & - \\
\hline $\begin{array}{l}\text { Relatedness - Intrinsic } \\
\text { values }\end{array}$ & .30 & .38 & $-2.66^{*}$ & - & - & - \\
\hline Autonomy - Job demands & -.16 & -.16 & 0.00 & - & - & - \\
\hline $\begin{array}{l}\text { Autonomy - Work-family } \\
\text { conflict }\end{array}$ & -.22 & -.27 & 1.37 & - & - & - \\
\hline $\begin{array}{l}\text { Competence - Job } \\
\text { demands }\end{array}$ & -.17 & -.04 & $-5.20 *$ & - & - & - \\
\hline $\begin{array}{l}\text { Relatedness - Job } \\
\text { demands }\end{array}$ & -.11 & -.04 & $-2.78^{*}$ & - & - & - \\
\hline Autonomy - Skill variety & .63 & .39 & $12.01^{*}$ & - & - & - \\
\hline $\begin{array}{l}\text { Autonomy - Job } \\
\text { autonomy }\end{array}$ & .42 & .70 & -19.90 & - & - & - \\
\hline
\end{tabular}


Table 8 (continued)

\begin{tabular}{|c|c|c|c|c|c|c|}
\hline \multirow[b]{2}{*}{ Effect } & \multicolumn{3}{|c|}{ Publication Status } & \multicolumn{3}{|c|}{ Scale Used } \\
\hline & $\rho_{\text {published }}$ & $\rho_{\text {unpublished }}$ & $Z$ & $\begin{array}{c}\rho_{\text {Deci, Ryan, Gagne, }} \\
\text { Leone, Usunov, \& } \\
\text { Kornazheva (2001) }\end{array}$ & $\begin{array}{c}\rho_{\text {Van den Broeck, }} \\
\text { Vansteenkiste, De Witte, } \\
\text { Soenens, \& Lens (2010) }\end{array}$ & $Z$ \\
\hline $\begin{array}{l}\text { Autonomy - Social } \\
\text { support }\end{array}$ & .31 & .48 & $-7.27^{*}$ & - & - & - \\
\hline $\begin{array}{l}\text { Competence - Skill } \\
\text { variety }\end{array}$ & .28 & .22 & $2.33^{*}$ & - & - & - \\
\hline $\begin{array}{l}\text { Competence - Job } \\
\text { autonomy }\end{array}$ & .16 & .27 & $-5.48^{*}$ & - & - & - \\
\hline $\begin{array}{l}\text { Competence - Social } \\
\text { support }\end{array}$ & .16 & .17 & -0.37 & - & - & - \\
\hline $\begin{array}{l}\text { Relatedness - Job } \\
\text { autonomy }\end{array}$ & .28 & .29 & -0.51 & - & - & - \\
\hline $\begin{array}{l}\text { Relatedness - Social } \\
\text { support }\end{array}$ & .51 & .56 & $-2.52 *$ & - & - & - \\
\hline $\begin{array}{l}\text { Autonomy - Leader } \\
\text { autonomy support }\end{array}$ & - & - & - & .50 & .52 & -0.56 \\
\hline $\begin{array}{l}\text { Autonomy - Perceived } \\
\text { organizational support }\end{array}$ & .61 & .72 & $-4.67^{*}$ & - & - & - \\
\hline $\begin{array}{l}\text { Autonomy - } \\
\text { Organizational exchange }\end{array}$ & .51 & .17 & $8.10^{*}$ & - & - & - \\
\hline $\begin{array}{l}\text { Autonomy - Fairness } \\
\text { perceptions }\end{array}$ & .40 & .41 & -0.35 & - & - & - \\
\hline $\begin{array}{l}\text { Competence - Leader } \\
\text { autonomy support }\end{array}$ & - & - & - & .35 & .29 & 1.39 \\
\hline $\begin{array}{c}\text { Competence }- \text { Perceived } \\
\text { organizational support }\end{array}$ & .39 & .53 & $-4.20 *$ & - & - & - \\
\hline $\begin{array}{l}\text { Competence - } \\
\text { Organizational exchange }\end{array}$ & .55 & .15 & $9.68 *$ & - & - & - \\
\hline $\begin{array}{l}\text { Competence - Fairness } \\
\text { perceptions }\end{array}$ & .11 & .09 & -1.33 & - & - & - \\
\hline $\begin{array}{l}\text { Relatedness - Leader } \\
\text { autonomy support }\end{array}$ & - & - & - & .41 & .34 & 1.68 \\
\hline $\begin{array}{r}\text { Relatedness - Perceived } \\
\text { organizational support }\end{array}$ & .52 & .55 & -0.99 & - & - & - \\
\hline $\begin{array}{l}\text { Relatedness - } \\
\text { Organizational exchange }\end{array}$ & .49 & .11 & $8.82 *$ & - & - & - \\
\hline $\begin{array}{l}\text { Relatedness - Fairness } \\
\text { perceptions }\end{array}$ & .43 & .35 & $2.79 *$ & - & - & - \\
\hline Autonomy - Engagement & .64 & .66 & $-2.72 *$ & .38 & .37 & 0.81 \\
\hline Autonomy - Strain & -.41 & -.43 & 0.93 & -.46 & -.45 & -0.36 \\
\hline Autonomy - Burnout & -.62 & -.59 & $-3.25^{*}$ & -.65 & -.60 & $-3.37 *$ \\
\hline $\begin{array}{r}\text { Competence - } \\
\text { Engagement }\end{array}$ & .43 & .33 & $9.20 *$ & .38 & .37 & 0.77 \\
\hline Competence - Strain & -.42 & -.29 & $-5.73 *$ & -.38 & -.36 & -0.66 \\
\hline Competence - Burnout & -.38 & -.23 & $-11.39 *$ & -.65 & -.24 & $-21.78^{*}$ \\
\hline Relatedness - Engagement & .49 & .46 & $3.06^{*}$ & .53 & .45 & $7.69^{*}$ \\
\hline Relatedness - Strain & -.41 & -.24 & $-7.17^{*}$ & -.35 & -.28 & $-2.36^{*}$ \\
\hline
\end{tabular}


Table 8 (continued)

\begin{tabular}{|c|c|c|c|c|c|c|}
\hline \multirow[b]{2}{*}{ Effect } & \multicolumn{3}{|c|}{ Publication Status } & \multicolumn{3}{|c|}{ Scale Used } \\
\hline & $\rho_{\text {published }}$ & $\rho_{\text {unpublished }}$ & $Z$ & $\begin{array}{c}\rho_{\text {Deci, Ryan, Gagne, }} \\
\text { Leone, Usunov, \& } \\
\text { Kornazheva (2001) }\end{array}$ & $\begin{array}{c}\rho_{\text {Van den Broeck, }} \\
\text { Vansteenkiste, De Witte, } \\
\text { Soenens, \& Lens (2010) }\end{array}$ & $Z$ \\
\hline Relatedness - Burnout & -.41 & -.37 & $-3.28 *$ & -.50 & -.36 & $-7.08^{*}$ \\
\hline $\begin{array}{l}\text { Autonomy - Job } \\
\text { satisfaction }\end{array}$ & .69 & .71 & $-2.19^{*}$ & .69 & .73 & $-2.96^{*}$ \\
\hline $\begin{array}{l}\text { Autonomy - Affective } \\
\text { commitment }\end{array}$ & .62 & .61 & 1.04 & .64 & .67 & $-3.11^{*}$ \\
\hline $\begin{array}{l}\text { Autonomy - Turnover } \\
\text { intentions }\end{array}$ & -.63 & -.12 & $-37.28^{*}$ & -.66 & -.13 & $-38.89 *$ \\
\hline $\begin{array}{l}\text { Competence - Job } \\
\text { satisfaction }\end{array}$ & .54 & .47 & $5.26^{*}$ & .71 & .29 & $21.55^{*}$ \\
\hline $\begin{array}{l}\text { Competence - Affective } \\
\text { commitment }\end{array}$ & .25 & .28 & $-2.10^{*}$ & .25 & .20 & $3.11^{*}$ \\
\hline $\begin{array}{l}\text { Competence - Turnover } \\
\text { intentions }\end{array}$ & -.16 & .02 & $-10.89^{*}$ & -.15 & .04 & $-11.23 *$ \\
\hline $\begin{array}{l}\text { Relatedness - Job } \\
\text { satisfaction }\end{array}$ & .55 & .50 & $3.86^{*}$ & .58 & .50 & $4.14 *$ \\
\hline $\begin{array}{l}\text { Relatedness - Affective } \\
\text { commitment }\end{array}$ & .63 & .55 & $7.99 *$ & .62 & .56 & $5.45^{*}$ \\
\hline $\begin{array}{l}\text { Relatedness - Turnover } \\
\text { intentions }\end{array}$ & -.45 & -.07 & -24.89 & -.43 & -.08 & -22.31 \\
\hline $\begin{array}{l}\text { Autonomy - Task } \\
\text { performance }\end{array}$ & .28 & .30 & -0.79 & .38 & .22 & $5.34 *$ \\
\hline $\begin{array}{l}\text { Autonomy - Proactive } \\
\text { performance }\end{array}$ & .37 & .33 & 1.66 & .38 & .28 & $3.68^{*}$ \\
\hline $\begin{array}{l}\text { Competence - Task } \\
\text { performance }\end{array}$ & .36 & .45 & -3.91 & .40 & .43 & -1.10 \\
\hline $\begin{array}{l}\text { Competence - Proactive } \\
\text { performance }\end{array}$ & .41 & .36 & $2.14^{*}$ & .40 & .29 & $4.10^{*}$ \\
\hline $\begin{array}{l}\text { Competence - Deviance } \\
\text { behavior }\end{array}$ & -.18 & -.32 & $4.24 *$ & -.37 & -.13 & -7.59 \\
\hline $\begin{array}{l}\text { Relatedness - Task } \\
\text { performance }\end{array}$ & .27 & .25 & 0.78 & .29 & .18 & $3.52 *$ \\
\hline $\begin{array}{l}\text { Relatedness - Proactive } \\
\text { performance }\end{array}$ & .37 & .36 & 0.42 & .36 & .31 & 1.85 \\
\hline $\begin{array}{l}\text { Relatedness - Deviance } \\
\text { behavior }\end{array}$ & - & - & - & -.30 & -.12 & $-5.56^{*}$ \\
\hline $\begin{array}{l}\text { Autonomy - External } \\
\text { motivation }\end{array}$ & -.15 & -.05 & $-5.58^{*}$ & - & - & - \\
\hline $\begin{array}{l}\text { Autonomy - Introjected } \\
\text { motivation }\end{array}$ & .12 & .04 & $3.90^{*}$ & - & - & - \\
\hline $\begin{array}{l}\text { Autonomy - Identified } \\
\text { motivation }\end{array}$ & .42 & .40 & 1.28 & .47 & .39 & $3.19 *$ \\
\hline $\begin{array}{l}\text { Autonomy - Intrinsic } \\
\text { motivation }\end{array}$ & .55 & .71 & -15.18 & - & - & - \\
\hline $\begin{array}{l}\text { Competence - External } \\
\text { motivation }\end{array}$ & -.10 & -.04 & $-3.33 *$ & - & - & - \\
\hline
\end{tabular}


Table 8 (continued)

\begin{tabular}{|c|c|c|c|c|c|c|}
\hline \multirow[b]{2}{*}{ Effect } & \multicolumn{3}{|c|}{ Publication Status } & \multicolumn{3}{|c|}{ Scale Used } \\
\hline & $\rho_{\text {published }}$ & $\rho_{\text {unpublished }}$ & $Z$ & $\begin{array}{c}\rho_{\text {Deci, Ryan, Gagne, }} \\
\text { Leone, Usunov, \& } \\
\text { Kornazheva (2001) }\end{array}$ & $\begin{array}{c}\rho_{\text {Van den Broeck, }} \\
\text { Vansteenkiste, De Witte, } \\
\text { Soenens, \& Lens (2010) }\end{array}$ & $Z$ \\
\hline $\begin{array}{l}\text { Competence - Introjected } \\
\text { motivation }\end{array}$ & .15 & .03 & $5.86^{*}$ & - & - & - \\
\hline $\begin{array}{l}\text { Competence - Identified } \\
\text { motivation }\end{array}$ & .37 & .28 & $5.42 *$ & .56 & .28 & $11.22 *$ \\
\hline $\begin{array}{l}\text { Competence - Intrinsic } \\
\text { motivation }\end{array}$ & .35 & .30 & $3.16^{*}$ & - & - & - \\
\hline $\begin{array}{l}\text { Relatedness - External } \\
\text { motivation }\end{array}$ & -.09 & .03 & $-6.64 *$ & - & - & - \\
\hline $\begin{array}{l}\text { Relatedness - Introjected } \\
\text { motivation }\end{array}$ & .13 & .04 & $4.39 *$ & - & - & - \\
\hline $\begin{array}{l}\text { Relatedness - Identified } \\
\text { motivation }\end{array}$ & .31 & .30 & 0.59 & .37 & .29 & $3.68^{*}$ \\
\hline $\begin{array}{l}\text { Relatedness - Intrinsic } \\
\text { motivation }\end{array}$ & .39 & .47 & $-5.55^{*}$ & - & - & - \\
\hline
\end{tabular}

Note: $\rho=$ estimate of corrected correlation; $Z=$ significant test of the difference between the corrected correlations. $* p<.05$.

(Pyszczynski, Greenberg, \& Solomon, 2000), our results indicate that the basic needs explained about half of the variance of negative aspects of well-being (i.e., negative affect and strain) as was explained of positive aspects of well-being (i.e., positive affect, general well-being, and engagement). Although there were exceptions (i.e., life satisfaction and burnout), the criticism does not appear to be entirely without merit. Along these lines, and perhaps most problematic for SDT, was the fact that the basic psychological needs did not explain any meaningful variance in external or introjected motivation. As with well-being, basic psychological needs seem to do a better job predicting more "positive" (identified and intrinsic) forms of motivation than more "negative" forms of motivation (external and introjected). These findings raise questions about the role of the needs at the start of the internalization process - when individuals move from external regulation to introjection - and run counter to SDT arguments that the satisfaction of each of the needs reduces external regulation (Deci \& Ryan, 2000). Applying a rigid interpretation of SDT's conceptualization of what is required for a construct to be considered a basic psychological need, these findings could be taken as disqualifying autonomy, competence, and relatedness as basic psychological needs. We return to this issue in our recommendations for future research.

\section{Should Needs for Autonomy, Competence, and Relatedness Be Averaged Together?}

We used three criteria to address whether it is appropriate to average needs for autonomy, competence, and relatedness together: whether the measures correlate strongly, whether the measures incrementally predict outcomes in RW analyses, and the nomological network of 
the antecedents of the three needs. Each of the three criteria suggests that it is not appropriate to average the three needs together or to use an overall need satisfaction score.

With respect to the first criterion, our analysis highlights that the needs can be highly correlated, though not so strongly (i.e., $\rho>.70$ ) as to indicate the needs are redundant with each other. With respect to the second criterion, the RW analyses indicate that each need generally predicts unique variance, meaning that averaging the measures is inappropriate. With respect to the third criterion, while the magnitude and direction of the relations between each need and any given correlate frequently appeared similar, the confidence intervals also often did not overlap. Although the confidence intervals overlapped more often than not, the number of nonoverlapping intervals was sizeable and support the notion that each need does not relate to all variables in an identical fashion. Given these differing nomological networks, it would be inappropriate to view the needs as equal and interchangeable.

Although the results should be interpreted with caution, another indication that averaging the measures is inappropriate is the fact that the RW analyses showed that competence occasionally predicted outcomes in the opposite direction from autonomy and relatedness. Specifically, competence led to less affective commitment, greater turnover intentions, and higher levels of absenteeism in the RW analyses. These results would seem to indicate that once the shared variance with autonomy and relatedness is accounted for, those who are highly competent may feel less tied to their place of work - presumably because they believe they could find employment elsewhere. Although such an explanation is plausible from a theoretical point of view, we again suggest caution against overinterpreting these results, as from a methodological point of view, they may be due to a suppression effect.

\section{Does the Measure Used Influence the Results?}

Finally, our examinations of whether the measure used moderated the findings produced mixed results. In general, the Deci et al. (2001) scale demonstrated stronger relations with outcomes than the Van den Broeck et al. (2010) scale, suggesting it may have greater predictive validity. However, two caveats should be mentioned: First, as noted previously, the stronger relations may be due to the Deci et al. scale incorporating consequences or antecedents of need satisfaction within its items; second, the number of studies available for comparisons between the two scales was often small. One exception to the latter point was the large number of studies available for comparison with respect to the relations among the needs themselves. Here, it is noteworthy that the relations between the needs were strongly affected by the measure used. In particular, correlations among the needs using the Deci et al. scale were quite strong for the relation of autonomy and competence $(\rho=.64)$ and competence and relatedness $(\rho=.50)$, compared to the same relations using the Van den Broeck et al. scale ( $\rho=.41$ and .30 , respectively). As the needs should (in theory) be relatively independent, the strong relation among the needs in the Deci et al. scale may be a concern (a point we return to below).

\section{Going Forward: Recommendations for Future Research}

As noted previously, taken as a whole, our findings can be viewed as generally supporting SDT's perspective on basic psychological needs while also highlighting both gaps and issues 
in the literature. We now turn to ways to address these gaps and issues, as well as providing recommendations for future SDT research drawn more holistically from our review of the literature on basic psychological need satisfaction at work.

\section{Recommendation 1: Begin Considering Need Satisfaction and Need Frustration}

One of the more critical findings of our meta-analysis was that satisfaction of basic needs did not substantively predict more negative forms of motivation (and, to a lesser extent, more negative forms of well-being). As noted previously, a strict interpretation of these findings for motivation would suggest that autonomy, competence, and relatedness are not basic psychological needs (though such a conclusion would need to be balanced against the largely positive findings seen otherwise). These findings regarding prediction of negative outcomes, however, are generally reminiscent of findings in other literatures (e.g., Colquitt, Long, Rodell, \& Halvorsen-Ganepola, 2015; Ferris, Johnson, Rosen, Djurdjevic, Chang, \& Tan, 2013 ) that the presence and absence of positive events, such as experiencing need satisfaction, are more associated with intense experience of positive outcomes, while the presence and absence of negative events, such as, we argue, need frustration, are more associated with intense experience of negative outcomes. That is, positive and negative events are not simply opposite ends of a spectrum, as the absence of a positive does not imply a negative and the absence of a negative does not imply a positive.

In other words, we believe that the relatively weaker effects linking need satisfaction to more negative outcomes does not indicate that basic psychological needs are irrelevant for these outcomes; rather, we advance that such effects are more likely to emerge when examining need frustration or thwarting. Within SDT, the focus has traditionally been on need satisfaction, not need frustration, and most measures do not distinguish between the two (Deci et al, 2001; Van den Broeck et al., 2010). However, to fully appreciate the effects of basic psychological needs, we believe that both need satisfaction and need frustration should be examined, as a few studies have tentatively begun to do (Bartholomew, Ntoumanis, Ryan, \& Thøgersen-Ntoumani, 2011; Gillet, Lafrenière, Vallerand, Huart, \& Fouquereau, 2014); we encourage such work and suggest it may be particularly useful for addressing the issues raised by our meta-analytic results.

\section{Recommendation 2: Be Mindful of the Measure Used (and How Needs Are Modeled)}

Our review suggests that the measure used may affect the results, with the Deci et al. (2001) measure generally showing stronger relations both between needs and antecedents/ outcomes and among the needs themselves. Although the former may be viewed as arguing for the criterion-related validity of the measure, the latter may be viewed as arguing against the psychometric properties of the measure, given that each need is supposed to be independent and unlikely to always co-occur (Deci \& Ryan, 1995). The Deci et al. measure demonstrated particularly strong corrected correlations between autonomy and competence and autonomy and relatedness, perhaps owing to the lower reliability observed for the autonomy scale in general (average $\alpha=.68$ ). We therefore suggest authors be mindful of the measures 
used in their study: Although adjustments to the Deci et al. measure may resolve these problems, from a psychometric perspective, the Van den Broeck et al. (2010) measure would seem to be more reliable and to demonstrate lower correlations among the needs (which is in line with what SDT would predict). Nevertheless - and per our first recommendation-we also encourage the development of new measures assessing both need satisfaction and frustration.

Our review also suggests ceasing the practice of combining the three basic needs into an overall scale and, instead, strengthens the argument that the needs are not interchangeable, cannot compensate for each other in an overall need satisfaction scale (Sheldon \& Niemiec, 2006), and are unlikely to always co-occur (Deci \& Ryan, 1995). Combining the needs into an overall need satisfaction measure runs contrary to SDT's conceptualization of the three basic needs as separate, noncompensatory entities. At the same time, we sympathize with authors who have done so, particularly because such overall measures are often justified in the face of the high correlations between the measures (Gagné, 2003) or the better fit of a confirmatory factor analysis that treats them as indicators of an overall construct (Rosen et al., 2014; Van den Broeck et al., 2008).

The practice of averaging the three need measures into an overall need satisfaction score is typically accompanied by using the three need measure scores as indicators of a latent overall need satisfaction factor in a structural equation model. From a methodological point of view, such broad latent factors can be appropriate and demonstrate predictive validity (although the extent to which the latent factors are substantive vs. simply an indicator of common method variance must be examined; R. E. Johnson, Rosen, \& Djurdjevic, 2011). However, from a theoretical point of view, it is unclear what such a latent construct would represent within SDT. This is not to say that latent overall need satisfaction constructs should never be used but, rather, that more empirical and theoretical work needs to be done to determine (a) whether it is appropriate to model SDT needs in this way and (b) what the overall construct represents, theoretically.

\section{Recommendation 3: Compare and Integrate SDT With Other Management Theories}

One of SDT's most interesting assumptions regarding basic psychological needs is that the three needs of autonomy, competence, and relatedness will consistently predict psychological growth, internalization, and well-being over and above the effects of any other possible need candidates (e.g., need for power), while other possible need candidates would not incrementally explain these outcomes. Unfortunately, few studies have tackled this prediction by comparing the effects of SDT needs on these outcomes versus other needs. To test this assumption, we need studies including the basic needs together with other candidate needs, as well as measures of psychological growth, internalization, and well-being (a prerequisite for testing this assumption).

The lack of research comparing basic psychological needs versus other possible need candidates represents a broader criticism that can be leveled at SDT (although it applies equally to the motivation literature as a whole): There has been relatively little comparative research pitting basic psychological needs not only against other needs but also against constructs from other motivational theories. That is, many motivation theories exist that do not 
posit specific needs but nevertheless posit motivational mechanisms that could be plausibly viewed as affecting psychological growth, internalization, and well-being. Although some exceptions exist (e.g., Lian et al., 2012, compared basic psychological need satisfaction against social exchange and justice theory constructs), by and large, comparative studies against other motivational theory constructs are infrequent. The solution here is straightforward: We need (no pun intended) more research comparing SDT's basic psychological needs against other potential needs and motivational constructs - in the prediction of both psychological growth, internalization, and well-being and other workplace outcomes. ${ }^{4}$

Aside from comparative tests of basic psychological needs against needs or constructs from other theories, another approach would be to integrate basic psychological need satisfaction within other theories - or, other theories within basic psychological need satisfaction. Along the lines of the latter, Rosen and colleagues (2014) examined what best predicted various behaviors (organizational citizenship behaviors, creativity, and proactivity) by comparing basic psychological need satisfaction with resource constructs from social exchange theory (Cropanzano \& Mitchell, 2005) and conservation of resources theory (Hobfoll, 1989) - two commonly used theories in organizational research. They argued that the "socioemotional" resources exchanged or "personal" resources conserved in (respectively) social exchange and conservation of resources theories simply represent satisfaction of basic psychological needs and, hence, that social exchange theory and conservation of resources theory can be viewed as essentially redundant with basic psychological needs theory. Across four studies, they found basic psychological needs predicted the outcomes typically associated with social exchange and conservation of resources theories (i.e., social exchange relationships and strain). Moreover, once the effects of basic psychological needs on outcomes were accounted for, exchange relationships and strain had little predictive validity on their own, which was interpreted as supporting basic psychological need theory as having greater utility than social exchange or conservation of resources theory.

Whether the research is comparative or integrative in nature, we believe more research aimed at examining basic psychological needs theory in comparison to other motivation theories should be one of the next steps for basic psychological needs research. The results of our meta-analysis clearly indicate that basic psychological needs have been linked to a wide variety of outcomes, and while new research linking it to other outcomes is certainly not discouraged, we believe this will not necessarily advance the position and respectability of basic psychological needs research within management. Rather, research demonstrating that basic psychological needs influence outcomes over and above constructs associated with other popular needs or constructs derived from other management theories or research demonstrating that other popular management theories can be subsumed within or otherwise integrated with basic psychological needs (per Rosen et al., 2014) would be the best way to advance the understanding of and attention to basic psychological needs research (and, by proxy, SDT as a whole).

\section{Recommendation 4: Make a Concerted Effort to Assess Cultural Boundary Conditions}

Although the satisfaction of the basic needs is argued to be universally beneficial (Ryan \& Deci, 2000), this view has been challenged (e.g., Markus \& Kitayama, 1991; Schwartz, 
2000). From a relativist perspective, it is believed that individuals mostly (if not only) benefit from the satisfaction of those needs that are explicitly valued in their culture. As collectivistic cultures value social relations and group membership more so than individualistic cultures (Oyserman, Coon, \& Kemmelmeier, 2002), employees from collectivistic cultures may therefore benefit more from the satisfaction of the need for relatedness, compared to employees from individualistic countries. In our meta-analysis, we could not provide a thorough test of the impact of culture as a moderator of the relations examined in our meta-analysis as too few studies have examined the effects of workplace need satisfaction in other cultures. ${ }^{5}$ This lack of cross-cultural research is surprising, particularly given the globalization of management research (Kirkman \& Law, 2005) and SDT's positioning as a universal theory of needs (Deci \& Ryan, 2000).

The solution here is again straightforward: More research in nonindividualistic cultures would be ideal. In examining the effect of culture, we encourage researchers to directly assess potentially relevant cultural differences (e.g., via measures designed to assess individualism/collectivism) as possible moderators of the effects of basic psychological needs. Moreover, such moderators need not be limited to cultural dimensions: Different cultures may also give rise to individual differences in the degree to which one values autonomy, competence, or relatedness. Although, as noted earlier, most SDT research does not focus on need strength, owing to the assumptions that basic psychological needs are innate, work on "the need for the basic needs" could complement cross-cultural research. Some attempts at assessing need strength have been made (Chen et al., 2015), but this work needs further development using validated measures to assess need strength suitable for an organizational context.

\section{Recommendation 5: Improve the Methods of SDT Studies}

Although not apparent in the results reported above, after our review of the SDT literature, two methodological aspects stood out to us. First, the vast majority of the data we reviewed used self-reported data to assess constructs; a slight exception to this trend was in respect to behaviors (e.g., performance, deviance, or citizenship behaviors), though even here the majority of studies used self-reported behaviors. For some outcomes (e.g., job satisfaction), this is perhaps unsurprising, and we are certainly not advocating that SDT researchers begin using non-self-report sources of data haphazardly (self-reports for need satisfaction and motivation are particularly appropriate; see Chan, 2009, for a discussion of the pros and cons of self-reports). Moreover, the reliance on self-report data is not new for management research. Yet at the same time, particularly given SDT's focus on well-being, we were surprised that more objective measures of short-term variations in health (e.g., physiological measures of the stress response, such as salivary cortisol readings and resting blood pressure; Ganster \& Rosen, 2013) or long-term consequences (e.g., metabolic system functioning, body mass index, or sleep quality) were not used more frequently. Beyond health outcomes, research linking basic psychological needs to other objective outcomes (e.g., turnover and profitability) would similarly be useful.

Second, the vast majority of the studies we examined used cross-sectional research designs (although, again, exceptions exist, primarily when examining behaviors). Taken together with the prior point, this suggests that the majority of the studies we examined were 
cross-sectional self-report studies. Owing to increased expectations among reviewers and editors, this type of study design is difficult (if not impossible) to publish in top management journals (Ashkanasy, 2010). Moreover, such designs are susceptible to alternate methodological interpretations (such as common method variance) and causal interpretations. We therefore discourage cross-sectional self-report studies dealing with basic psychological needs and strongly encourage SDT researchers to incorporate time-separated or longitudinal/ cross-lagged designs into their studies, including also studies at the daily level tapping into within-person fluctuations of need satisfaction (for more, see Podsakoff, MacKenzie, Lee, \& Podsakoff, 2003).

\section{Conclusion}

In line with early 20th-century researchers, we believe human needs are an essential part of motivation, and SDT certainly is one of the more comprehensive theories of basic psychological needs. Our review of SDT's conceptualization of basic psychological needs and the empirical support for the theory is largely good news for SDT researchers: The needs for autonomy, competence, and relatedness mostly fit the criteria set out for what basic psychological needs represent. At the same time, we found qualifications to these conclusions and also found that some of the more interesting - and controversial - aspects of SDT research await more comprehensive testing in organizational settings. Our hope is that through further research in line with our recommendations, SDT and the area of basic psychological needs will continue to flourish and influence organizational thinking in the years ahead.

\section{Notes}

1. Although the broader SDT framework has been reviewed in management journals before (Gagné \& Deci, 2005; Sheldon, Turban, Brown, Barrick, \& Judge, 2003), these reviews have been primarily designed to introduce SDT concepts to organizational audiences. Perhaps most critically, these reviews have been narrative in nature and have not focused specifically on basic psychological needs.

2. Aside from identified motivation, Deci and Ryan (2000) also proposed integrated motivation as being the most autonomous form of motivation (short of intrinsic motivation). While identified motivation refers to seeing the importance of the extrinsically motivated behavior, integrated motivation was proposed to assess fully incorporating the reasons underlying the behavior in one's sense of self. However, to date, no research has demonstrated that integrated motivation accounts for additional variance in outcomes after including identified or intrinsic motivation, and studies (and questionnaires) now typically no longer assess this type of motivation separately but, rather, incorporate it with the assessment of identification (Gagné et al, 2015). In line with this, this review assesses identified but not integrated motivation.

3. As a result of rounding (e.g., from .004 to .00 or from -.004 to -.00 ), we considered a confidence interval to include 0 only if the confidence interval ranged from -.00 (or lower) to a positive value or from .00 (or higher) to a negative value. Thus, for example, a confidence interval ranging from -.17 to .00 would be classified as including 0 , while a confidence interval ranging from .17 to .00 would not be classified as including 0 (similarly, a confidence interval ranging from -.17 to -.00 would not include 0 , while a confidence interval ranging from -.17 to .00 would include 0$)$.

4. In the online supplemental material, we provide the results (see Table S11) of an RW analysis examining the relative effects of psychological need satisfaction vis-à-vis job security - which could be considered a proxy for Maslow's (1943) need for security in the context of work - in the prediction of job satisfaction, affective commitment, turnover intentions, and task performance. Although our results are limited by a small number of studies $(k=3)$ relating job security to the three basic psychological needs, our results provide some initial evidence that job security can explain additional variance in job satisfaction, affective commitment, turnover intentions, and task performance. 
5. Although (as noted) the analysis is limited by a small number of studies examining need satisfaction in other cultures, in the online supplemental material, we provide tables examining culture as a possible moderator of our findings. Where possible, the online supplemental material also examines other possible methodological moderators (e.g., time-lagged vs. cross-sectional study designs; self- vs. supervisor vs. peer ratings for the criterion, e.g., performance).

\section{References}

Arthur, W., Bennett, W., \& Huffcutt, A. I. 2001. Conducting meta-analysis using SAS. Mahwah, NJ: Erlbaum.

Ashkanasy, N. M. 2010. Publishing today is more difficult than ever. Journal of Organizational Behavior, 31 : 1-3.

Baard, P. P., Deci, E. L., \& Ryan, R. M. 2004. Intrinsic need satisfaction: A motivational basis of performance and well-being in two work settings. Journal of Applied Social Psychology, 34: 2045-2068.

Bandura, A. 1977. Self-efficacy: Toward a unifying theory of behavioral change. Psychological Review, 84: 191-215.

Bartholomew, K. J., Ntoumanis, N., Ryan, R. M., \& Thøgersen-Ntoumani, C. 2011. Psychological need thwarting in the sport context: Assessing the darker side of athletic experience. Journal of Sport \& Exercise Psychology, 33: $75-102$.

Baumeister, R. F., \& Leary, M. R. 1995. The need to belong: Desire for interpersonal attachments as a fundamental human motivation. Psychological Bulletin, 117: 497-529.

Chan, D. 2009. So why ask me? Are self-report data really that bad? In C. E. Lance \& R. J. Vandenberg (Eds.), Statistical and methodological myths and urban legends: Received doctrine, verity, and fable in the organizational and social sciences: 311-338. New York: Routledge.

Chen, B., Vansteenkiste, M., Beyers, W., Boone, L., Deci, E. L., Van der Kaap-Deeder, J., Duriez, B., Lens, W., Matos, L., Mouratidis, A., Ryan, R. M., Sheldon, K. M., Soenens, B., Van Petegem, S., \& Verstuyf, J. 2015. Basic psychological need satisfaction, need frustration, and need strength across four cultures. Motivation and Emotion, 39: 216-236.

Cheung, S. F., \& Chan, D. K. S. 2004. Dependent effect sizes in meta-analysis: Incorporating the degree of interdependence. Journal of Applied Psychology, 89: 780-791.

Colquitt, J. A., Long, D. M., Rodell, J. B., \& Halvorsen-Ganepola, M. D. K. 2015. Adding the "in" to justice: A qualitative and quantitative investigation of the differential effects of justice rule adherence and violation. Journal of Applied Psychology, 100: 278-297.

Cortina, J. M. 2003. Apples and oranges (and pears, oh my!): The search for moderators in meta-analysis. Organizational Research Methods, 6: 415-439.

Crawford, E. R., Lepine, J. A., \& Rich, B. L. 2010. Linking job demands and resources to employee engagement and burnout: A theoretical extension and meta-analytic test. Journal of Applied Psychology, 95: 834-848.

Cropanzano, R., \& Mitchell, M. S. 2005. Social exchange theory: An interdisciplinary review. Journal of Management, 31: 874-900.

deCharms, R. 1968. Personal causation: The internal affective determinants of behaviour. New York: Academic Press.

Deci, E. L., \& Ryan, R. M. 1980. The empirical exploration of intrinsic motivational processes. In L. Berkowitz (Ed.), Advances in experimental social psychology, vol. 13: 39-80. New York: Academic Press.

Deci, E. L., \& Ryan, R. M. 1985. Intrinsic motivation and self-determination in human behavior. New York: Plenum.

Deci, E. L., \& Ryan, R. M. 1995. Human autonomy: The basis for true self-esteem. In M. Kernis (Ed.), Efficacy, agency and self-esteem: 31-49. New York: Plenum.

Deci, E. L., \& Ryan, R. M. 2000. The "what" and "why" of goal pursuits: Human needs and the self-determination of behavior. Psychological Inquiry, 11: 227-268.

Deci, E. L., Ryan, R. M., Gagne, M., Leone, D. R., Usunov, J., \& Kornazheva, B. P. 2001. Need satisfaction, motivation, and well-being in the work organizations of a former Eastern Bloc country: A cross-cultural study of self-determination. Personality and Social Psychology Bulletin, 27: 930-942.

Deci, E. L., Ryan, R. M., \& Koestner, R. 1999. A meta-analytic review of experiments examining the effects of extrinsic rewards on intrinsic motivation. Psychological Bulletin, 125: 627-668.

Diefendorff, J. M., \& Chandler, M. M. 2011. Motivating employees. In S. Zedeck (Ed.), APA handbook of industrial and organizational psychology, vol. 3: Maintaining, expanding and contracting the organization: 65-135. Washington, DC: American Psychological Association. 
Edmunds, J., Ntoumanis, N., \& Duda, J. L. 2006. A test of self-determination theory in the exercise domain. Journal of Applied Social Psychology, 36: 2240-2265.

Ferris, D. L., Johnson, R. E., Rosen, C. C., Djurdjevic, E., Chang, C.-H., \& Tan, J. A. 2013. When is success not satisfying? Integrating regulatory focus and approach/avoidance motivation theories to explain the relation between core self-evaluation and job satisfaction. Journal of Applied Psychology, 98: 342-353.

Fugate, M., Kinicki, A. J., \& Ashforth, B. E. 2004. Employability: A psycho-social construct, its dimensions, and applications. Journal of Vocational Behavior, 65: 14-38.

Gagné, M. 2003. The role of autonomy support and autonomy orientation in prosocial behavior engagement. Motivation and Emotion, 27: 199-223.

Gagné, M., \& Deci, E. L. 2005. Self-determination theory and work motivation. Journal of Organizational Behavior, 26: 331-362.

Gagné, M., Forest, J., Vansteenkiste, M., Crevier-Braud, L., Van den Broeck, A., Aspeli, A. K., Bellerose, J., Benabou, C., Chemolli, E., Güntert, S. T., Halvari, H., Indiyastuti, D. L., Johnson, P. A., Molstad, M. H., Naudin, M., Ndao, A., Olafsen, A. H., Roussel, P., Wang, Z., \& Westbye, C. 2015. The Multidimensional Work Motivation Scale: Validation evidence in seven languages and nine countries. European Journal of Work and Organizational Psychology, 24: 178-196.

Ganster, D. C., \& Rosen, C. C. 2013. Work stress and employee health: A multi-disciplinary review. Journal of Management, 39: 1085-1122.

Gillet, N., Lafrenière, M.-A. K., Vallerand, R. J., Huart, I., \& Fouquereau, E. 2014. The effects of autonomous and controlled regulation of performance-approach goals on well-being: A process model. British Journal of Social Psychology, 53: 154-174.

Greguras, G. J., \& Diefendorff, J. M. 2009. Different fits satisfy different needs: Linking person-environment fit to employee commitment and performance using self-determination theory. Journal of Applied Psychology, 94: 465-477.

Greguras, G. J., \& Diefendorff, J. M. 2010. Why does proactive personality predict employee life satisfaction and work behaviors? A field investigation of the mediating role of the self-concordance model. Personnel Psychology, 63: 539-560.

Hall, S. M., \& Brannick, M. T. 2002. Comparison of two random-effects methods of meta-analysis. Journal of Applied Psychology, 87: 377-389.

Hinkin, T. R. 1998. A brief tutorial on the development of measures for use in survey questionnaires. Organizational Research Methods, 1: 104-121.

Hobfoll, S. E. 1989. Conservation of resources: A new attempt at conceptualizing stress. American Psychologist, 44: 513-524.

Hogan, R. 1998. Reinventing personality. Journal of Social and Clinical Psychology, 17: 1-10.

Hull, C. L. 1943. Principles of behavior: An introduction to behavior theory. New York: Appleton-CenturyCrofts.

Hunter, J. E., \& Schmidt, F. L. 2004. Methods of meta-analysis: Correcting error and bias in research findings. Thousand Oaks, CA: Sage.

Ilardi, B. C., Leone, D., Kasser, T., \& Ryan, R. M. 1993. Employee and supervisor ratings of motivation: Main effects and discrepancies associated with job satisfaction and adjustment in a factory setting. Journal of Applied Social Psychology, 23: 1789-1805.

Johnson, J. W. 2000. A heuristic method for estimating the relative weight of predictor variables in multiple regression. Multivariate Behavioral Research, 35: 1-19.

Johnson, R. E., Rosen, C. C., \& Djurdjevic, E. 2011. Assessing the impact of common method variance on higher order multidimensional constructs. Journal of Applied Psychology, 96: 744-761.

Kanfer, R. 1990. Motivation theory and industrial/organizational psychology. In M. D. Dunette \& L. Hough (Eds.), Handbook of industrial and organizational psychology, vol. 1: Theory in industrial and organizational psychology: 75-170. Palo Alto, CA: Consulting Psychologists Press.

Kirkman, B. L., \& Law, K. S. 2005. International management research in $A M J$ : Our past, present, and future. Academy of Management Journal, 48: 738.

Latham, G. P., \& Pinder, C. C. 2005. Work motivation theory and research at the dawn of the twenty-first century. Annual Review of Psychology, 56: 485-516.

LeBreton, J. M., \& Tonidandel, S. 2008. Multivariate relative importance: Extending relative weight analysis to multivariate criterion spaces. Journal of Applied Psychology, 93: 329-345. 
Lian, H., Ferris, D. L., \& Brown, D. J. 2012. Does taking the good with the bad make things worse? How abusive supervision and leader-member exchange interact to impact need satisfaction and organizational deviance. Organizational Behavior and Human Decision Processes, 117: 41-52.

Markus, H. R., \& Kitayama, S. 1991. Culture and the self: Implications for cognition, emotion, and motivation. Psychological Review, 98: 224-253.

Maslow, A. 1943. A theory of human motivation. Psychological Review, 50: 370-396.

McClelland, D. C. 1965. Achievement and entrepreneurship: A longitudinal study. Journal of Personality and Social Psychology, 14: 389-392.

Moran, C. M., Diefendorff, J. M., Kim, T.-Y., \& Liu, Z.-Q. 2012. A profile approach to self-determination theory motivations at work. Journal of Vocational Behavior, 81: 354-363.

Murray, H. A. 1938. Explorations in personality. New York: Oxford University Press.

Ng, J. Y. Y., Ntoumanis, N., Thogersen-Ntoumani, C., Deci, E. L., Ryan, R. M., Duda, J. L., \& Williams, G. C. 2012. Self-determination theory applied to health contexts: A meta-analysis. Perspectives on Psychological Science, 7: 325-340.

Nunnally, J. C. 1967. Psychometric theory. New York: McGraw Hill.

Nunnally, J. C., \& Bernstein, I. H. 1994. Psychometric theory (3rd ed.). New York: McGraw-Hill.

Oyserman, D., Coon, H. M., \& Kemmelmeier, M. 2002. Rethinking individualism and collectivism: Evaluation of theoretical assumptions and meta-analyses. Psychological Bulletin, 128: 3-72.

Pinder, C. 2008. Work motivation in organizational behavior (2nd ed.). New York: Psychology Press.

Podsakoff, P. M., MacKenzie, S. B., Lee, J.-Y., \& Podsakoff, N. P. 2003. Common method biases in behavioral research: A critical review of the literature and recommended remedies. Journal of Applied Psychology, 88: 879-903.

Pyszczynski, T., Greenberg, J., \& Solomon, S. 2000. Toward a dialectical analysis of growth and defensive motives. Psychological Inquiry, 11: 301-305.

Rosen, C. C., Ferris, D. L., Brown, D. J., Chen, Y., \& Yan, M. 2014. Perceptions of organizational politics: A need satisfaction paradigm. Organization Science, 25: 1026-1055.

Rosenthal, R. 1979. The file drawer problem and tolerance for null results. Psychological Bulletin, 86: 638-641.

Rosenthal, R. 1991. Meta-analytic procedures for social research (rev. ed.). Newbury Park, CA: Sage.

Ryan, R. M., \& Deci, E. L. 2000. Self-determination theory and the facilitation of intrinsic motivation, social development, and well-being. American Psychologist, 55: 68-78.

Ryan, R. M., \& Deci, E. L. 2001. On happiness and human potentials: A review of research on hedonic and eudemonic well-being. Annual Review of Psychology, 52: 141-166.

Sagie, A., \& Koslowsky, M. 1993. Detecting moderators with meta-analysis: An evaluation and comparison of techniques. Personnel Psychology, 46: 629-640.

Schwartz, B. 2000. Self-determination: The tyranny of freedom. American Psychologist, 55: 79-88.

Sheldon, K. M., Elliot, A. J., Kim, Y., \& Kasser, T. 2001. What is satisfying about satisfying events? Testing 10 candidate psychological needs. Journal of Personality and Social Psychology, 80: 325-339.

Sheldon, K. M., \& Kasser, T. 1998. Pursuing personal goals: Skills enable progress, but not all progress is beneficial. Personality and Social Psychology Bulletin, 24: 1319-1331.

Sheldon, K. M., \& Niemiec, C. P. 2006. It's not just the amount that counts: Balanced need satisfaction also affects well-being. Journal of Personality and Social Psychology, 91: 331-341.

Sheldon, K. M., Turban, D. B., Brown, K. G., Barrick, M. R., \& Judge, T. A. 2003. Applying self-determination theory to organizational research. Research in Personnel and Human Resource Management, 22: 357-393.

Steers, R. M., Mowday, R. T., \& Shapiro, D. L. 2004. Introduction to special topic forum: The future of work motivation theory. Academy of Management Review, 29: 379-387.

Trougakos, J. P., Hideg, I., Cheng, B. H., \& Beal, D. J. 2013. Lunch breaks unpacked: The role of autonomy as a moderator of recovery during lunch. Academy of Management Journal, 57: 405-421.

Van den Broeck, A., Lens, W., De Witte, H., \& Van Coillie, H. 2013. Unraveling the importance of the quantity and the quality of workers' motivation for well-being: A person-centered perspective. Journal of Vocational Behavior, 82: 69-78.

Van den Broeck, A., Vansteenkiste, M., De Witte, H., \& Lens, W. 2008. Explaining the relationships between job characteristics, burnout, and engagement: The role of basic psychological need satisfaction. Work \& Stress, 22: $277-294$. 
Van den Broeck, A., Vansteenkiste, M., De Witte, H., Soenens, B., \& Lens, W. 2010. Capturing autonomy, competence, and relatedness at work: Construction and initial validation of the Work-Related Basic Need Satisfaction scale. Journal of Occupational and Organizational Psychology, 83: 981-1002.

Vansteenkiste, M., Lens, W., \& Deci, E. L. 2006. Intrinsic versus extrinsic goal contents in self-determination theory: Another look at the quality of academic motivation. Educational Psychologist, 41: 19-31.

Vansteenkiste, M., \& Ryan, R. M. 2013. On psychological growth and vulnerability: Basic psychological need satisfaction and need frustration as a unifying principle. Journal of Psychotherapy Integration, 23: 263-280.

White, R. 1959. Motivation reconsidered: The concept of competence. Psychological Review, 66: 279-333.

Whitener, E. M. 1990. Confusion of confidence intervals and credibility intervals in meta-analysis. Journal of Applied Psychology, 75: 315-321. 